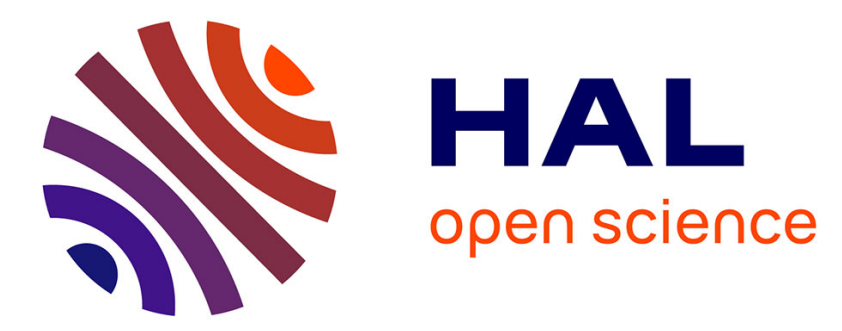

\title{
Comparison of nonlocal continualization schemes for lattice beams and plates
}

\author{
F. Hache, Noël Challamel, I. Elishakoff, C. M. Wang
}

\section{To cite this version:}

F. Hache, Noël Challamel, I. Elishakoff, C. M. Wang. Comparison of nonlocal continualization schemes for lattice beams and plates. Archive of Applied Mechanics, 2017, 87 (7), pp.1105-1138. 10.1007/s00419-017-1235-z . hal-01693911

\section{HAL Id: hal-01693911 \\ https://hal.science/hal-01693911}

Submitted on 31 Dec 2019

HAL is a multi-disciplinary open access archive for the deposit and dissemination of scientific research documents, whether they are published or not. The documents may come from teaching and research institutions in France or abroad, or from public or private research centers.
L'archive ouverte pluridisciplinaire HAL, est destinée au dépôt et à la diffusion de documents scientifiques de niveau recherche, publiés ou non, émanant des établissements d'enseignement et de recherche français ou étrangers, des laboratoires publics ou privés. 


\title{
F. Hache · N. Challamel · I. Elishakoff - C. M. Wang \\ Comparison of nonlocal continualization schemes for lattice beams and plates
}

\begin{abstract}
This paper investigates both stability and vibration of nonlocal beams or plates in the presence of compressive forces. Various nonlocal structural models have been proposed to capture the inherent scale effects of lattice-based beams or plates. These nonlocal models are either based on continualization of the difference equations of the original lattice problem (labeled as continualized nonlocal models), or developed from phenomenological nonlocal approaches such as Eringen's type nonlocality. Considered herein are several continualization schemes that lead to either fourth or sixth order spatial governing differential or partial differential equation. Even if the new continualized nonlocal plate models differ in their mathematical description, they appear to furnish very close macroscopic results as shown from asymptotic expansion arguments. The continualized nonlocal beam and plate models and the phenomenological approaches are also introduced from variational arguments. The key role of boundary conditions is shown especially for Eringen's nonlocal model that is not necessarily variationally based. For each of them, the buckling load and the natural frequencies are determined for simply supported beams and plates and compared to their counterparts obtained from the lattice model. The small length scale coefficient of the nonlocal beam or plate models is intrinsically constant and problem independent for the continualized approaches, whereas it is calibrated for the phenomenological models based on the equivalence with the reference microstructured model and consequently, depends on the load, the buckling or vibration mode or the aspect ratio. It is found that the nonlocal continualized approaches are more efficient than the nonlocal phenomenological ones. For beam problems, continualized nonlocal and phenomenological approaches such as Eringen's nonlocal theory can become the same. However, for plate problems, phenomenological approaches may differ significantly from continualized nonlocal ones; thereby offering one the opportunity to have a new class of two-dimensional nonlocal models.
\end{abstract}

F. Hache $\cdot$ N. Challamel $(\bowtie)$

UBS - IRDL, Centre de Recherche, Université de Bretagne Sud, University of South Brittany (UBS),

Rue de Saint Maudé, BP 92116, 56321 Lorient Cedex, France

E-mail: noel.challamel@univ-ubs.fr

F. Hache

E-mail: florian.hache@univ-ubs.fr; fhache2014@fau.edu

I. Elishakoff

Department of Ocean and Mechanical Engineering, Florida Atlantic University, Boca Raton, FL 33481-0991, USA

E-mail: elishako@fau.edu

C. M. Wang

School of Civil Engineering, University of Queensland, St Lucia, QLD 4067, Australia

E-mail: cm.wang@uq.edu.au 
Keywords Nonlocal elasticity · Continualization procedure · Padé approximants · Beam · Plate · Buckling · Vibration · Lattice

\section{Introduction}

The advent of nanomaterials in the last decade has spurred tremendous interest in the scientific community to study small scale structures. At a finer scale, the dimensions of the structure are close to those of its material microstructure such as grain size or lattice spacing between two successive atoms at the atomic scale. The continuum aspect of the material at this scale is questionable due to the occurrence of some microstructural effects. Nonlocal continuum theories, however, can be used to capture the discreteness of the material at the subscale. A basic difference between local and nonlocal theories is the definition of the stress at a certain point. The stress is only a function of strain at that point in local mechanics but it is potentially a function of strains at all points in nonlocal mechanics [1]. The first nonlocal mechanics investigations have been carried out in the 1960s by Krumhansl [2,3], Rogula [4], Eringen [5,6], Kröner and Datta [7], Kunin [8], mostly for crystal applications, leading to integro-differential equations to be solved.

A basic approach to justify the use of nonlocal models is to consider whether the structure is represented by discrete systems that are composed of finite number of cells. For instance, Hencky [9] investigated the mechanical behavior of Bernoulli-Euler beams by considering a chain of rigid segmented beams connected by elastic rotational springs. The theoretical link between discrete and continuous systems is not new and has already been established a few centuries ago by Lagrange [10] who showed that a one-dimensional structure (mainly a string or a rod) is asymptotically equivalent to a lattice system with an infinite number of links (see also the recent discussion of this problem in [11]. A few decades later, Piola constructed nonlocal continuous models such as peridynamics models from discrete interactions [12,13]. Lattice models are basic physical systems that can accurately represent fundamental interactions at the microscale. Nowadays, numerical solutions of lattice-based equations may be readily solved with the aid of modern computers.

In 1983, Eringen [14] proposed a nonlocal stress gradient elasticity model to describe the mechanical behavior of lattice-based macrostructure. This nonlocal model may be classified as a phenomenological approach and is based on a postulated nonlocal relationship between the stress and the strain, while the equilibrium equations are be kept in a local form. This nonlocal law is dependent on a dimensionless material constant $e_{0}$ associated with the discreteness of the matter at the subscale. The length scale parameter of the nonlocal model may be calibrated from the Born-Kármán [15] lattice model for axial wave propagation [14]. Even though Eringen's theory is an efficient phenomenological nonlocal model and it has been applied to many structural configurations, this theory has been shown to have some limitations in some particular cases. For instance, the nonlocal effect does not affect a nonlocal beam in the presence of a concentrated load [16,17], where the solution is identical to the local counterpart. This issue has been investigated specifically in $[16,17]$ where it has been shown that one can capture some scale effects, for general loading by adopting a combination of the local and nonlocal strain measures. Moreover, in the nonlocal Eringen's model [14], the nonlocal effect affects the mechanical behavior of the structure through the constant $e_{0}$, which is supposed to be independent of the load. The value of this constant was found to be equal to 0.39 by fitting the wave dispersive curves with those of the Born-Karman theory for axial lattices [14]. Lattice beams or plates can also be investigated for calibrating the length scale coefficient $e_{0}$ of nonlocal beams or plates, as recently performed by different researchers [18-20]. They showed that the small length scale coefficient $e_{0}$ lies between $1 / \sqrt{12}$ for beam buckling problem and $1 / \sqrt{6}$ for beam vibration problem, leading to a paradoxical result because it meant that the constant coefficient apparently varies with respect to applied load and physics of the problem. Thus, to solve the apparent length scale-dependent paradox, alternative nonlocal models have been proposed in order to keep a constant small length scale coefficient based on micromechanics arguments. The objective is to develop continuous equations from discrete ones. To this end, among the available methodologies, two basic methods can be distinguished, namely the homogenization of the discrete media and the continualization of lattice models $([21,22]$ for instance).

The continualization process is based on the possible equivalence between continuous nonlocal model and discrete lattice model [23]. Mathematically, the problem is equivalent to the approximation of lattice difference operator by continuous differential ones. The pseudodifferential operator associated with the lattice problem is expanded by using the Taylor series [24] or rational expansion. Padé approximants have been introduced by Rosenau [25,26] following the works of Benjamin et al. [27] and Collins [28] for axial lattices. Since then, they have been widely used in the literature [20,29]. In these approaches, the accuracy of the model depends 
on the order of the asymptotic expansion. The objective is to develop continuous equations that contain the characteristic properties of a discrete structure [23]. Continuous equations based on lattice mechanics lead to governing differential equations in which the small length scale coefficient is associated with a length having a physical meaning, namely the internal characteristic length of the lattice spacing for such one-dimensional bending systems (or beam-like models). Andrianov et al [30] used this technique to approximate the dynamics behavior of a monoatomic and a biatomic axial chain with continuum models. This continualization process has been already applied to lattice beams (also called Hencky chain model) [19] and lattice plates [18,20]. Giorgio et al [31] recently developed continuous approximation of Hencky chain model with nonlinear elastic interaction, thus asymptotically leading to a nonlinear-type Euler-Bernoulli beam model.

In a recent paper, Challamel et al. [20] developed a continualized plate model based on the Padé approximant of the uncountered pseudodifferential operators and a phenomenological model called hybrid continualized model. In contrast to the usual Eringen's based phenomenological nonlocal plate models leading to a governing differential equation with fourth-order derivative, the continualized models introduced in [20] contains a new sixth-order spatial derivative terms in the governing differential equation. Consequently, in these continualized models, the solution requires additional boundary conditions, and this new constraint raises the issue of the meaning of these higher-order boundary conditions. Nonlinear nonlocal vibrations of beams (based on Eringen's differential model) are studied by Ansari and Hemmatnezhad [32] by finite elements, whereas the nonlocal nonlinear vibrations of buckled double-layered nanoplates (also based on Eringen's differential model) are studied by Wang et al. [33] using a Galerkin's procedure based on a double-mode truncation.

The aims of this study are: (a) to propose lattice beam and plate models for the microstructured elastic system that covers bending, buckling and vibration phenomena, (b) to develop new continualized and phenomenological models and to investigate the possible equivalence between the two approaches and possible links between two continualized models, (c) to establish the exact solutions associated with the newly proposed lattice-based nonlocal models and compare them against the exact lattice solutions, and (d) to establish the superiority of one of the models over the others. In comparing the different models, the small length scale coefficient, which is calibrated for the phenomenological models, will be compared and the influence of different parameters such as the buckling and vibration mode shapes or the aspects ratios on the small length scale coefficient will be discussed for the different models.

\section{Presentation of models for nonlocal beam}

We start from the lattice beam model in elasticity. First, we present the model for a beam that takes into account an axial load and also include a dynamic framework. The four associated nonlocal continua will be investigated next. They will be referred to as the fourth-order continualized model, its sixth-order derivative counterpart, the phenomenological fourth-order derivative and the phenomenological sixth-order derivative.

\subsection{Lattice beams}

The microstructured beam-grid model, also called the lattice beam model, is taken as the reference model or lattice-based solution for calibration at a later stage. The essential idea underlying the microstructured beam models is to replace the curved lines of deformation by straight lines [9]. In this discrete representation, the bending moments are lumped at places that rotations are localized [34]. Following this idea, a microstructured beam-grid for modeling can be reproduced by an assembly of chain net systems of rigid straight elements connected at frictionless joints where rotations are localized as shown in Fig. 1.

So, consider a lattice beam of length $L$, modeled by $n$ rigid segmented beams of length $a(L=n a)$ and concentrated inertia mass $m$ connected by elastic rotational springs of stiffness $C=E I / a$ with $E$ is the Young modulus and $I$ the second moment of area of the beams, as shown in Fig. 1. The entire microstructured column is axially loaded by a compressive concentrated load $P$. We define $m_{0}$ such as $m_{0}=m / a$ and $\omega$ the frequency of the beam. At the $i$ th node, the deflection is $w_{i}$ and the bending moment is $M_{i}$.

In the lattice model, the beam is governed by the following moment curvature relationship and the equation of motion 

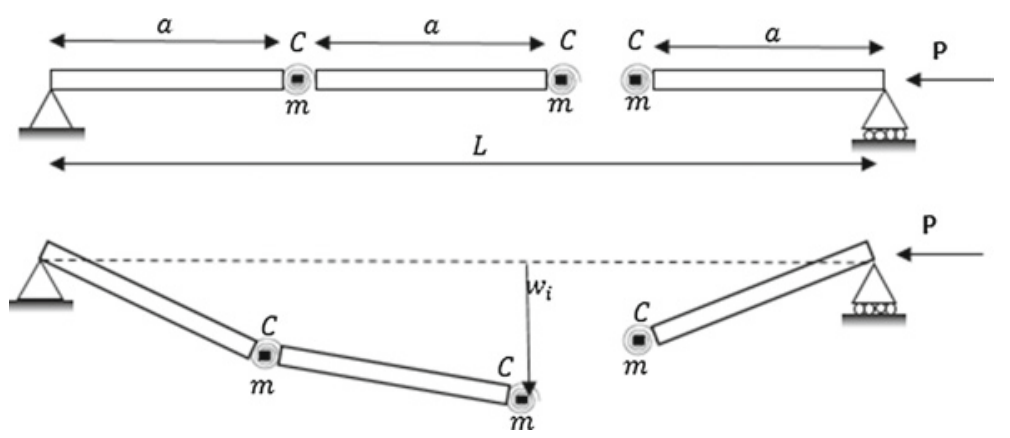

Fig. 1 Microstructured beam model

$$
\begin{aligned}
M_{i} & =E I\left(\frac{w_{i+1}-2 w_{i}+w_{i-1}}{a^{2}}\right) \\
\frac{M_{i+1}-2 M_{i}+M_{i-1}}{a^{2}} & =-P \frac{w_{i+1}-2 w_{i}+w_{i-1}}{a^{2}}+m_{0} \omega^{2} w_{i}
\end{aligned}
$$

Equations (1) and (2) are the finite difference formulation of the continuous Bernoulli-Euler beam equations as given by

$$
\begin{gathered}
M=E I \frac{\mathrm{d}^{2} w}{\mathrm{~d} x^{2}} \\
P \frac{\mathrm{d}^{2} w}{\mathrm{~d} x^{2}}+\frac{\mathrm{d}^{2} M}{\mathrm{~d} x^{2}}-m_{0} \omega^{2} w=0
\end{gathered}
$$

Equations (1) and (2) lead to a linear fourth-order difference equation for the microstructured beam-grid model

$$
P\left(\frac{w_{i+1}-2 w_{i}+w_{i-1}}{a^{2}}\right)+E I\left(\frac{w_{i+2}-4 w_{i+1}+6 w_{i}-4 w_{i-1}+w_{i-2}}{a^{4}}\right)-m_{0} \omega^{2} w_{i}=0
$$

This difference equation is the finite difference formulation of the continuous governing differential equation of the Bernoulli-Euler model:

$$
E I \frac{\mathrm{d}^{2} w}{\mathrm{~d} x^{4}}-m_{0} \omega^{2} w+P \frac{\mathrm{d}^{2} w}{\mathrm{~d} x^{2}}=0
$$

For simply supported ends, the exact solution of the lattice problem is assumed to be

$$
w_{i}=w_{0} \sin \left(\frac{m \pi i}{n}\right)
$$

We set the critical buckling load and the fundamental natural frequency of the continuous Bernoulli-Euler column [35]:

$$
P_{E}=E I\left(\frac{\pi}{L}\right)^{2} ; \omega_{E}^{2}=\frac{E I}{m_{0}}\left(\frac{\pi}{L}\right)^{4}
$$

The substitution of Eq. (7) into Eq. (5) furnishes the following exact buckling load [19,35-38]:

$$
\lambda_{\text {(micro) }}=\min _{m}\left[\frac{2 n}{\pi} \sin \left(\frac{m \pi}{2 n}\right)\right]^{2}
$$

where $\lambda=P / P_{E}$ is the nondimensional buckling load parameter. In the case of free vibration, the natural frequency is also given by substituting Eq. (7) into Eq. (5), and it is [39,40]:

$$
\Omega_{(\text {micro })}^{2}=\left[\frac{2 n}{\pi} \sin \left(\frac{m \pi}{2 n}\right)\right]^{4}
$$

where $\Omega=\omega / \omega_{E}$ is the nondimensional natural frequency parameter. 


\subsection{Fourth-order continualized model}

Next, we consider the discrete representation of the continuum displacement fields. It involves $w_{i}=w\left(x_{i}\right)=$ $w(x)$, where $w_{i}$ and $w$ are the displacements of the discrete and the equivalent continuous systems, respectively, whereas the displacements of the neighboring particles $w_{i \pm 1}$ are replaced by $w\left(x_{i} \pm a\right)$. By applying Taylor series to the difference operators that describe discrete particle interactions, the following continualization process is applied for sufficiently smooth variations of the displacement function:

$$
\begin{gathered}
w_{i}=w\left(x_{i}\right)=w(x) \\
w_{i \pm 1}=w\left(x_{i} \pm a\right)=\sum_{k=0}^{\infty}( \pm 1)^{k} \frac{a^{k}}{k !} \frac{\mathrm{d}^{k}}{\mathrm{~d} x^{k}} w(x)=\left[e^{ \pm a \frac{\mathrm{d}}{\mathrm{d} x}}\right][w]
\end{gathered}
$$

Thus, the second derivative under the finite difference form is reduced to a pseudodifferential operator:

$$
\frac{w_{i+1}-2 w_{i}+w_{i-1}}{a^{2}}=\frac{1}{a^{2}} \sum_{k=0}^{\infty} 2 \frac{a^{2 k}}{(2 k) !} \frac{\mathrm{d}^{2 k}}{\mathrm{~d} x^{2 k}} w(x)=\frac{4}{a^{2}} \sinh ^{2}\left(\frac{a}{2} \frac{\mathrm{d}}{\mathrm{d} x}\right) w(x)
$$

The Taylor expansion of the pseudodifferential operator at the second order provides:

$$
\frac{4}{a^{2}} \sinh ^{2}\left(\frac{a}{2} \frac{\mathrm{d}}{\mathrm{d} x}\right)=\frac{\mathrm{d}^{2}}{\mathrm{~d} x^{2}}\left[1+\frac{a^{2}}{12} \frac{\mathrm{d}^{2}}{\mathrm{~d} x^{2}}\right] w(x)+o\left(a^{2}\right)
$$

The second term represents the effect due to discreteness, with $a$ being the trace of the microscopic system. The pseudodifferential operator may be developed by means of the Padé approximation and:

$$
\frac{w_{i+1}-2 w_{i}+w_{i-1}}{a^{2}}=\frac{4}{a^{2}} \sinh ^{2}\left(\frac{a}{2} \frac{\mathrm{d}}{\mathrm{d} x}\right) w(x)=\frac{\frac{\mathrm{d}^{2}}{\mathrm{~d} x^{2}}}{1-\frac{a^{2}}{12} \frac{\mathrm{d}^{2}}{\mathrm{~d} x^{2}}} w+o\left(a^{2}\right)
$$

In a similar manner,

$$
\frac{w_{i+2}-4 w_{i+1}+6 w_{i}-4 w_{i-1}+w_{i-2}}{a^{2}}=\frac{16}{a^{4}} \sinh ^{4}\left(\frac{a}{2} \frac{\mathrm{d}}{\mathrm{d} x}\right) w(x)=\frac{\frac{\mathrm{d}^{4}}{\mathrm{~d} x^{4}}}{\left(1-\frac{a^{2}}{12} \frac{\mathrm{d}^{2}}{\mathrm{~d} x^{2}}\right)^{2}} w+o\left(a^{2}\right)
$$

The substitution of Eqs. (11), (15) and (16) into Eq. (5) leads to the continualized model based on pseudodifferential operators:

$$
\frac{16}{a^{4}} E I \sinh ^{4}\left(\frac{a}{2} \frac{\mathrm{d}}{\mathrm{d} x}\right) w+P \frac{4}{a^{2}} \sinh ^{2}\left(\frac{a}{2} \frac{\mathrm{d}}{\mathrm{d} x}\right) w-m_{0} \omega^{2} w=0
$$

which may be approximated by using rational expansion of these operators:

$$
E I \frac{\frac{\mathrm{d}^{4}}{\mathrm{~d} x^{4}}}{\left(1-\frac{a^{2}}{12} \frac{\mathrm{d}^{2}}{\mathrm{~d} x^{2}}\right)^{2}} w+P \frac{\frac{\mathrm{d}^{2}}{\mathrm{~d} x^{2}}}{1-\frac{a^{2}}{12} \frac{\mathrm{d}^{2}}{\mathrm{~d} x^{2}}} w-m_{0} \omega^{2} w+o\left(a^{2}\right)=0
$$

Multiplying Eq. (18) by $\left[1-\left(a^{2} / 12\right)\left(\mathrm{d}^{2} / \mathrm{d} x^{2}\right)\right]^{2}$, we obtain

$$
E I \frac{\mathrm{d}^{4} w}{\mathrm{~d} x^{4}}+P\left[1-\frac{a^{2}}{12} \frac{\mathrm{d}^{2}}{\mathrm{~d} x^{2}}\right] \frac{\mathrm{d}^{2} w}{\mathrm{~d} x^{2}}-m_{0} \omega^{2}\left[1-\frac{a^{2}}{6} \frac{\mathrm{d}^{2}}{\mathrm{~d} x^{2}}+\frac{a^{4}}{144} \frac{\mathrm{d}^{4}}{\mathrm{~d} x^{4}}\right] w+o\left(a^{4}\right)=0
$$

At the second order, it is consistent to neglect the fourth-order term in $a$ that is underlined in Eq. (19). Thus, it yields:

$$
E I \frac{\mathrm{d}^{4} w}{\mathrm{~d} x^{4}}+P\left[1-\frac{a^{2}}{12} \frac{\mathrm{d}^{2}}{\mathrm{~d} x^{2}}\right] \frac{\mathrm{d}^{2} w}{\mathrm{~d} x^{2}}-m_{0} \omega^{2}\left[1-\frac{a^{2}}{6} \frac{\mathrm{d}^{2}}{\mathrm{~d} x^{2}}\right] w=0
$$


This equation coincides with the one obtained by Challamel et al. [35]. Furthermore, this equation can be equivalently obtained by continualization of the discrete constitutive law and the discrete equilibrium equation. Indeed, the discrete moment-curvature relation in Eq. (1) may be expressed using the second-order pseudodifferential operator, i.e.

$$
M=\frac{4 E I}{a^{2}} \sinh ^{2}\left(\frac{a}{2} \frac{\mathrm{d}}{\mathrm{d} x}\right) w=E I \frac{\frac{\mathrm{d}^{2}}{\mathrm{~d} x^{2}}}{1-\frac{a^{2}}{12} \frac{\mathrm{d}^{2}}{\mathrm{~d} x^{2}}} w+o\left(a^{2}\right)
$$

whereas the equilibrium equation Eq. (2) is given by:

$$
\begin{aligned}
& \frac{4}{a^{2}} \sinh ^{2}\left(\frac{a}{2} \frac{\mathrm{d}}{\mathrm{d} x}\right) M+P \frac{4}{a^{2}} \sinh ^{2}\left(\frac{a}{2} \frac{\mathrm{d}}{\mathrm{d} x}\right) w-m_{0} \omega^{2} w \\
& =\frac{\frac{\mathrm{d}^{2}}{\mathrm{~d} x^{2}}}{1-\frac{a^{2}}{12} \frac{\mathrm{d}^{2}}{\mathrm{~d} x^{2}}} M+P \frac{\frac{\mathrm{d}^{2}}{\mathrm{~d} x^{2}}}{1-\frac{a^{2}}{12} \frac{\mathrm{d}^{2}}{\mathrm{~d} x^{2}}} w-m_{0} \omega^{2} w+o\left(a^{2}\right)=0
\end{aligned}
$$

Multiplying Eqs. (21) and (22) by $\left[1-\left(a^{2} / 12\right)\left(\mathrm{d}^{2} / \mathrm{d} x^{2}\right)\right]$, we obtain the differential approximations:

$$
\begin{aligned}
M-\frac{a^{2}}{12} \frac{\mathrm{d}^{2} M}{\mathrm{~d} x^{2}} & =E I \frac{\mathrm{d}^{2} w}{\mathrm{~d} x^{2}}+o\left(a^{2}\right) \\
\frac{\mathrm{d}^{2} M}{\mathrm{~d} x^{2}} & =-P \frac{\mathrm{d}^{2} w}{\mathrm{~d} x^{2}}+m_{0} \omega^{2} w-\frac{a^{2}}{12} m_{0} \omega^{2} \frac{\mathrm{d}^{2} w}{\mathrm{~d} x^{2}}+o\left(a^{2}\right)
\end{aligned}
$$

Note that the equilibrium equation is no longer local with this formulation. The substitution of Eq. (24) into Eq. (23) furnishes

$$
M=E I \frac{\mathrm{d}^{2} w}{\mathrm{~d} x^{2}}-\frac{P a^{2}}{12} \frac{\mathrm{d}^{2} w}{\mathrm{~d} x^{2}}+m_{0} \omega^{2} \frac{a^{2}}{12} w-\frac{a^{4}}{144} m_{0} \omega^{2} \frac{\mathrm{d}^{2} w}{\mathrm{~d} x^{2}}+o\left(a^{4}\right)
$$

We define a small length scale coefficient $e_{0}$ such as $e_{0}=1 / \sqrt{12}$. This coefficient is constant and structurally independent. The substitution of Eq. (25) into Eq. (24) leads to:

$$
E I \frac{\mathrm{d}^{4} w}{\mathrm{~d} x^{4}}+P\left[1-\left(e_{0} a\right)^{2} \frac{\mathrm{d}^{2}}{\mathrm{~d} x^{2}}\right] \frac{\mathrm{d}^{2} w}{\mathrm{~d} x^{2}}-m_{0} \omega^{2}\left[1-2\left(e_{0} a\right)^{2} \frac{\mathrm{d}^{2}}{\mathrm{~d} x^{2}}+\left(e_{0} a\right)^{4} \frac{\mathrm{d}^{4}}{\mathrm{~d} x^{4}}\right] w+o\left(a^{4}\right)=0
$$

This equation coincides with Eq. (19) and so by neglecting the higher-order term in $a$, it yields Eq. (20). Thus, the two continualizations lead to the same governing differential equation. There is an equivalence between continualization of the final governing equations and continualization of both the constitutive law and the equilibrium equations.

Furthermore, Eq. (20) may be derived from variational approach by considering the following bending strain energy $U$, the kinetic energy $T$ and the work $W$ done by the axial force $P$

$$
\begin{aligned}
U(w) & =\int_{0}^{L} \frac{1}{2} E I\left(\frac{\mathrm{d}^{2} w}{\mathrm{~d} x^{2}}\right)^{2} \mathrm{~d} x \\
T(w) & =\int_{0}^{L} \frac{1}{2} m_{0} \omega^{2}\left[w^{2}+\frac{a^{2}}{6}\left(\frac{\mathrm{d} w}{\mathrm{~d} x}\right)^{2}\right] \mathrm{d} x \\
W(w) & =\int_{0}^{L} \frac{1}{2} P\left[\left(\frac{\mathrm{d} w}{\mathrm{~d} x}\right)^{2}+\frac{a^{2}}{12}\left(\frac{\mathrm{d}^{2} w}{\mathrm{~d} x^{2}}\right)^{2}\right] \mathrm{d} x
\end{aligned}
$$

Thus, by applying the variational principle $\delta(U-T-W)$ based on Hamilton's principle, one gets Eq. (20) with the boundary conditions

$$
\begin{array}{r}
{\left[\left\{\left[E I-P \frac{a^{2}}{12}\right] \frac{\mathrm{d}^{3} w}{\mathrm{~d} x^{3}}+\left[P+m_{0} \omega^{2} \frac{a^{2}}{6}\right] \frac{\mathrm{d} w}{\mathrm{~d} x}\right\} \delta w\right]_{0}^{L}=0} \\
{\left[\left\{\left[E I-P \frac{a^{2}}{12}\right] \frac{\mathrm{d}^{2} w}{\mathrm{~d} x^{2}}\right\} \delta\left(\frac{\mathrm{d} w}{\mathrm{~d} x}\right)\right]_{0}^{L}=0}
\end{array}
$$




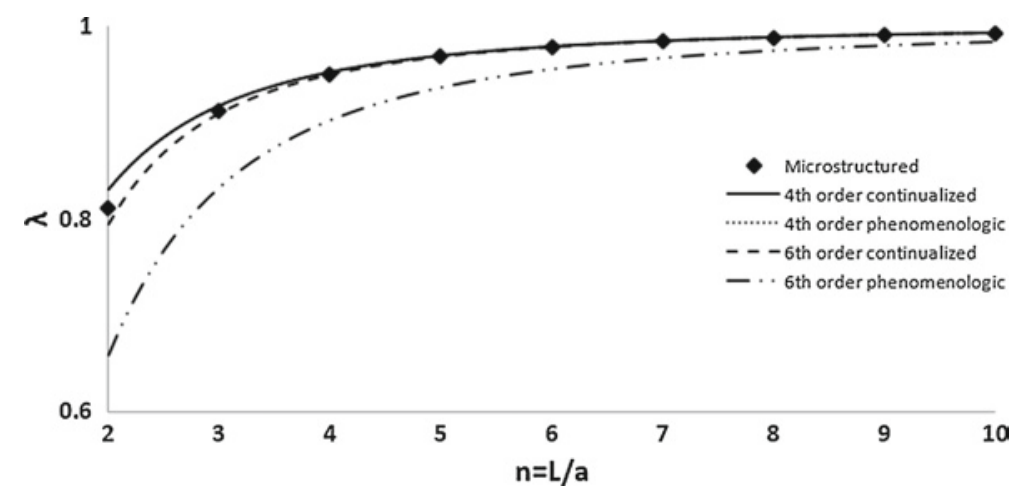

Fig. 2 Nondimensional critical buckling load for a beam considering the microstructured models the fourth- and sixth-order continualized model, the fourth- and sixth-order phenomenological models

These nonlocal boundary conditions clearly differ from the local ones, with additional terms related to the lattice spacing.

The solution is provided for a simply supported beam. For such boundary conditions, the solution may be expressed as:

$$
w=w_{0} \sin \left(\frac{m \pi x}{L}\right)
$$

where $m$ is an integer. By substituting Eq. (32) into Eq. (20), we obtain

$$
m^{4}-\lambda\left[1+\left(\frac{e_{0} a m \pi}{L}\right)^{2}\right] m^{2}-\bar{\Omega}^{2}\left[1+2\left(\frac{e_{0} a m \pi}{L}\right)^{2}\right]=0
$$

In statics $(\omega=0)$, the nondimensional buckling load parameter is (see Fig. 2):

$$
\lambda=\min _{m} m^{2} \frac{1}{1+\left(\frac{e_{0} a m \pi}{L}\right)^{2}}=\frac{1}{1+\left(\frac{e_{0} a \pi}{L}\right)^{2}}
$$

In free vibration $(P=0)$, the nondimensional frequency parameter is:

$$
\Omega^{2}=m^{2} \frac{1}{1+2\left(\frac{e_{0} a m \pi}{L}\right)^{2}}
$$

The variation of the nondimensional natural frequency parameter with respect to the number of elementary beam element is given in Fig. 3 for $m=1$.

\subsection{Sixth-order continualized model}

We suggest to use another continualization of the local constitutive law and the equilibrium equation. Indeed, we now use the following Taylor expansion:

$$
\frac{4}{a^{2}} \sinh ^{2}\left(\frac{a}{2} \frac{\mathrm{d}}{\mathrm{d} x}\right)=\frac{\mathrm{d}^{2}}{\mathrm{~d} x^{2}}\left(1+\frac{a^{2}}{12} \frac{\mathrm{d}^{2}}{\mathrm{~d} x^{2}}\right)+o\left(a^{2}\right)
$$

The substitution of Eq. (36) into Eq. (21) leads to

$$
M=E I\left[\frac{\mathrm{d}^{2} w}{\mathrm{~d} x^{2}}+\frac{a^{2}}{12} \frac{\mathrm{d}^{4} w}{\mathrm{~d} x^{4}}+o\left(a^{2}\right)\right]
$$

while we keep the nonlocal equilibrium equation based on the Padé approximants

$$
\frac{\frac{\mathrm{d}^{2}}{\mathrm{~d} x^{2}}}{1-\frac{a^{2}}{12} \frac{\mathrm{d}^{2}}{\mathrm{~d} x^{2}}} M=-P \frac{\frac{\mathrm{d}^{2}}{\mathrm{~d} x^{2}}}{1-\frac{a^{2}}{12} \frac{\mathrm{d}^{2}}{\mathrm{~d} x^{2}}} w+m_{0} \omega^{2} w+o\left(a^{2}\right)
$$




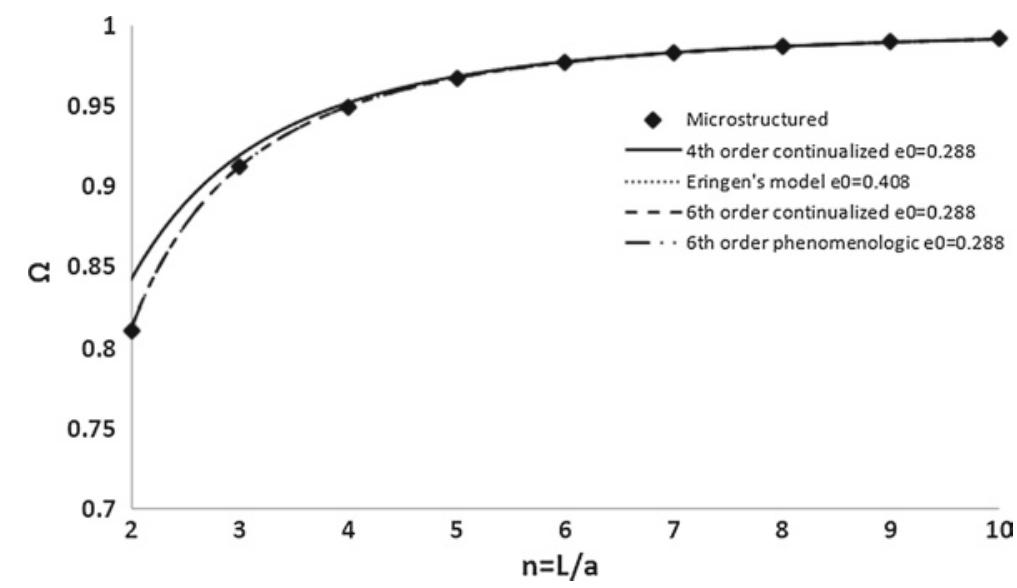

Fig. 3 Nondimensional vibration frequency for a beam considering the microstructured models the fourth- and sixth-order continualized model, the fourth- and sixth-order phenomenological models

The substitution of Eq. (37) into Eq. (38) and multiplying by $\left[1-\left(a^{2} / 12\right)\left(\mathrm{d}^{2} / \mathrm{d} x^{2}\right)\right]$ yields

$$
E I\left[1+\frac{a^{2}}{12} \frac{\mathrm{d}^{2}}{\mathrm{~d} x^{2}}+\underline{o\left(a^{2}\right)}\right] \frac{\mathrm{d}^{4} w}{\mathrm{~d} x^{4}}+P \frac{\mathrm{d}^{2} w}{\mathrm{~d} x^{2}}-m_{0} \omega^{2}\left[1-\frac{a^{2}}{12} \frac{\mathrm{d}^{2}}{\mathrm{~d} x^{2}}\right] w+o\left(a^{2}\right)=0
$$

Choosing for the fourth-order continualized model $e_{0}=1 / \sqrt{12}$ and by neglecting the underlined term of higher-order in $a$ leads to

$$
E I\left[1+\left(e_{0} a\right)^{2} \frac{\mathrm{d}^{2}}{\mathrm{~d} x^{2}}\right] \frac{\mathrm{d}^{4} w}{\mathrm{~d} x^{4}}+P \frac{\mathrm{d}^{2} w}{\mathrm{~d} x^{2}}-m_{0} \omega^{2}\left[1-\left(e_{0} a\right)^{2} \frac{\mathrm{d}^{2}}{\mathrm{~d} x^{2}}\right] w=0
$$

The new continualized governing equation is a sixth-order differential equation, instead of a fourth-order for the local Euler-Bernoulli one. Thus, the order of the governing differential equation can be increased by using a different continualization process. The two continualization methods presented herein are both valid but one requires the satisfaction of two additional boundary conditions for this sixth-order continualized model. It is worth noting that Eq. (40) may be asymptotically obtained from Eq. (20). Indeed, by multiplying Eq. (20) with $\left[1+\left(a^{2} / 12\right)\left(\mathrm{d}^{2} / \mathrm{d} x^{2}\right)\right]$, we obtain

$$
\begin{aligned}
& E I\left[1+\left(e_{0} a\right)^{2} \frac{\mathrm{d}^{2}}{\mathrm{~d} x^{2}}\right] \frac{\mathrm{d}^{4} w}{\mathrm{~d} x^{4}}+P\left[1-\left(e_{0} a\right)^{4} \frac{\mathrm{d}^{2}}{\mathrm{~d} x^{2}}\right] \frac{\mathrm{d}^{2} w}{\mathrm{~d} x^{2}} \\
& -m_{0} \omega^{2}\left[1-\left(e_{0} a\right)^{2} \frac{\mathrm{d}^{2}}{\mathrm{~d} x^{2}}-2\left(e_{0} a\right)^{4} \frac{\mathrm{d}^{4}}{\mathrm{~d} x^{4}}\right] w+o\left(a^{4}\right)=0
\end{aligned}
$$

Neglecting the underlined higher-order term in Eq. (41) leads to Eq. (40). In an equivalent manner, by applying the Padé approximant for the constitutive law and the Taylor approximant for the equation of motion:

$$
\begin{aligned}
\left(1-\frac{a^{2}}{12} \frac{\mathrm{d}^{2}}{\mathrm{~d} x^{2}}\right) M & =E I \frac{\mathrm{d}^{2} w}{\mathrm{~d} x^{2}}+o\left(a^{2}\right) \\
\frac{\mathrm{d}^{2}}{\mathrm{~d} x^{2}}\left[1+\frac{a^{2}}{12} \frac{\mathrm{d}^{2}}{\mathrm{~d} x^{2}}\right] M & =-P \frac{\mathrm{d}^{2}}{\mathrm{~d} x^{2}}\left[1+\frac{a^{2}}{12} \frac{\mathrm{d}^{2}}{\mathrm{~d} x^{2}}\right] w+m_{0} \omega^{2} w+o\left(a^{2}\right)
\end{aligned}
$$

Multiplying Eq. (43) by $\left[1-\left(a^{2} / 12\right)\left(\mathrm{d}^{2} / \mathrm{d} x^{2}\right)\right]$ and substituting Eq. (42) and assuming $e_{0}=1 / \sqrt{12}$, we obtain

$$
E I\left[1+\frac{a^{2}}{12} \frac{\mathrm{d}^{2}}{\mathrm{~d} x^{2}}\right] \frac{\mathrm{d}^{4}}{\mathrm{~d} x^{4}}+P \frac{\mathrm{d}^{2}}{\mathrm{~d} x^{2}}\left[1-\frac{a^{4}}{144} \frac{\mathrm{d}^{4}}{\mathrm{~d} x^{4}}\right] w-m_{0} \omega^{2}\left[1-\frac{a^{2}}{12} \frac{\mathrm{d}^{2}}{\mathrm{~d} x^{2}}\right] w+o\left(a^{4}\right)=0
$$

Neglecting the underlined term of higher-order in $a$ leads to Eq. (40). Reciprocally, Eq. (20) is obtained from Eq. (40) by multiplying by $\left[1-\left(e_{0} a\right)^{2}\left(\mathrm{~d}^{2} / \mathrm{d} x^{2}\right)\right]$ and neglecting the higher-order term in $a$. Although there is 
a link between Eqs. (20) and (40) through the differential operator $\left[1 \pm\left(e_{0} a\right)^{2}\left(\mathrm{~d}^{2} / \mathrm{d} x^{2}\right)\right]$, the two equations are not equivalent. Indeed, Eq. (40) being a sixth-order space derivative differential equation requires six boundary conditions (three at each end of the beam), whereas Eq. (20) only needs four boundary conditions. Thus, Eq. (40) involves the introduction of two additional boundary conditions.

Equation (41) may be also derived variationally by considering the energies $U, T$ and $W$ as follows

$$
\begin{gathered}
U(w)=\int_{0}^{L} \frac{1}{2} E I\left[\left(\frac{\mathrm{d}^{2} w}{\mathrm{~d} x^{2}}\right)^{2}-\frac{a^{2}}{12}\left(\frac{\mathrm{d}^{3} w}{\mathrm{~d} x^{3}}\right)^{2}\right] \mathrm{d} x \\
T(w)=\int_{0}^{L} \frac{1}{2} m_{0} \omega^{2}\left[w^{2}+\frac{a^{2}}{12}\left(\frac{\mathrm{d} w}{\mathrm{~d} x}\right)^{2}\right] \mathrm{d} x \\
W(w)=\int_{0}^{L} \frac{1}{2} P\left(\frac{\mathrm{d} w}{\mathrm{~d} x}\right)^{2} \mathrm{~d} x
\end{gathered}
$$

In the sixth-order continualized model, the work done by the force $P$ is not affected by the nonlocal effect. By applying the Hamilton principle, we obtain Eq. (40) with the natural boundary conditions

$$
\begin{gathered}
{\left[\left\{E I \frac{a^{2}}{12} \frac{\mathrm{d}^{5} w}{\mathrm{~d} x^{5}}+E I \frac{\mathrm{d}^{3} w}{\mathrm{~d} x^{3}}+\left[P+m_{0} \omega^{2} \frac{a^{2}}{12}\right] \frac{\mathrm{d} w}{\mathrm{~d} x}\right\} \delta w\right]_{0}^{L}=0} \\
{\left[\left\{E I \frac{a^{2}}{12} \frac{\mathrm{d}^{4} w}{\mathrm{~d} x^{4}}+E I \frac{\mathrm{d}^{2} w}{\mathrm{~d} x^{2}}\right\} \delta\left(\frac{\mathrm{d} w}{\mathrm{~d} x}\right)\right]_{0}^{L}=0} \\
{\left[\left\{E I \frac{a^{2}}{12} \frac{\mathrm{d}^{3} w}{\mathrm{~d} x^{3}}\right\} \delta\left(\frac{\mathrm{d}^{2} w}{\mathrm{~d} x^{2}}\right)\right]_{0}^{L}=0}
\end{gathered}
$$

The sixth-order space derivative model introduces two additional boundary conditions, one at each end (see Eq. (50) for the higher-order boundary conditions). A consequent difficulty would be to choose the appropriate boundary conditions. For a simply supported beam, at the boundaries, and assuming that the curvature vanishes at the boundary for the higher-order boundary condition, leads to:

$$
w=0 ; \frac{d^{4} w}{d x^{4}}=0 ; \frac{d^{2} w}{d x^{2}}=0
$$

The solution is provided for a simply supported beam. By substituting Eq. (32) into Eq. (40), we obtain

$$
\left[1-\left(\frac{e_{0} a m \pi}{L}\right)^{2}\right] m^{4}-\lambda m^{2}-\Omega^{2}\left[1+\left(\frac{e_{0} a m \pi}{L}\right)^{2}\right]=0
$$

In statics $(\omega=0)$, the nondimensional buckling load parameter is (see Fig. 2):

$$
\lambda=\min _{m}\left[m^{2}-\left(\frac{e_{0} a m^{2} \pi}{L}\right)^{2}\right]=1-\left(\frac{e_{0} a \pi}{L}\right)^{2}
$$

In free vibration $(P=0)$, the nondimensional frequency parameter is:

$$
\Omega^{2}=m^{2} \frac{1-\left(\frac{e_{0} a m \pi}{L}\right)^{2}}{1+\left(\frac{e_{0} a m \pi}{L}\right)^{2}}
$$

The variation of the nondimensional natural frequency parameter with respect to the number of elementary beam element is given in Fig. 3 for $m=1$.

The natural frequency and buckling load are not the same between the two continualized models. Indeed, the fourth-order continualized model is based on the Padé approximants applied to the pseudodifferential operator involved in the constitutive law and in the equilibrium equation, whereas the sixth-order continualized model is based on the use of Taylor approximant inn the constitutive law or in the equilibrium equation. This introduces a slight difference between both continualized nonlocal models, as shown in Fig. 3. Even if mathematically not strictly equivalent, both models give close results, for the order of magnitude considered. 


\subsection{Fourth-order phenomenological model: the Eringen's approach}

The phenomenological Eringen's nonlocal model is considered for capturing the scale effects associated with the Hencky lattice chain model or its continualized nonlocal counterpart. This nonlocal model is coupled to local-type equilibrium equations. First of all, we propose the following moment-curvature relation for the Eringen's model [14] applied at the beam scale [41,42]:

$$
\left[1-\left(e_{0} a\right)^{2} \frac{\mathrm{d}^{2}}{\mathrm{~d} x^{2}}\right] M=E I \frac{\mathrm{d}^{2}}{\mathrm{~d} x^{2}} w
$$

In contrast to the continualized models, the small length scale coefficient $e_{0}$ is unknown and has to be calibrated in order to match the results of the discrete model. Multiplying the continuous local equilibrium Eq. (4) by $\left[1-\left(e_{0} a\right)^{2}\left(\mathrm{~d}^{2} / \mathrm{d} x^{2}\right)\right]$ and substituting Eq. (55), we obtain the following governing differential equation $[43,44]$

$$
E I \frac{\mathrm{d}^{4} w}{\mathrm{~d} x^{4}}+P\left[1-\left(e_{0} a\right)^{2} \frac{\mathrm{d}^{2}}{\mathrm{~d} x^{2}}\right] \frac{\mathrm{d}^{2} w}{\mathrm{~d} x^{2}}-m_{0} \omega^{2}\left[1-\left(e_{0} a\right)^{2} \frac{\mathrm{d}^{2}}{\mathrm{~d} x^{2}}\right] w=0
$$

Equation (56) coincides with the governing differential equation obtained by Reddy [43] and Reddy and Pang [44].

This differential equation is close to Eq. (20) but the nonlocal term associated with the dynamic effect is different. In the fourth-order phenomenological model, the term associated with the dynamic effect is the same one associated with the static effect. Thus, it is not possible to make a perfect coincidence between the continualized nonlocal model and Eringen's nonlocal beam model, except in the pure buckling case $\omega=0$, or in the pure vibration case $P=0$. Consequently, the calibrated small length scale coefficient of Eringen's nonlocal model would be different in dynamics and statics and so, is structural problem dependent.

Furthermore, Eq. (56) may be obtained variationally by considering the energies $U, T$ and $W$ given by

$$
\begin{gathered}
U(w)=\int_{0}^{L} \frac{1}{2} E I\left(\frac{\mathrm{d}^{2} w}{\mathrm{~d} x^{2}}\right)^{2} \mathrm{~d} x \\
T(w)=\int_{0}^{L} \frac{1}{2} m_{0} \omega^{2}\left[w^{2}+\left(e_{0} a\right)^{2}\left(\frac{\mathrm{d} w}{\mathrm{~d} x}\right)^{2}\right] \mathrm{d} x \\
W(w)=\frac{1}{2} \int_{0}^{L} P\left[\left(\frac{\mathrm{d} w}{\mathrm{~d} x}\right)^{2}+\left(e_{0} a\right)^{2}\left(\frac{\mathrm{d}^{2} w}{\mathrm{~d} x^{2}}\right)^{2}\right] \mathrm{d} x
\end{gathered}
$$

These variational terms and the natural boundary conditions are given by Adali for the buckling problem [45] and the vibration problem [46]. ditions

By applying the variational principle $\delta(U-T-W)$, we obtain Eq. (56) with the natural boundary con-

$$
\begin{gathered}
{\left[\left\{\left[E I-P\left(e_{0} a\right)^{2}\right] \frac{\mathrm{d}^{3} w}{\mathrm{~d} x^{3}}+\left[P+m_{0} \omega^{2}\left(e_{0} a\right)^{2}\right] \frac{\mathrm{d} w}{\mathrm{~d} x}\right\} \delta w\right]_{0}^{L}=0} \\
{\left[\left\{\left[E I-P\left(e_{0} a\right)^{2}\right] \frac{\mathrm{d}^{2} w}{\mathrm{~d} x^{2}}\right\} \delta\left(\frac{\mathrm{d} w}{\mathrm{~d} x}\right)\right]_{0}^{L}=0}
\end{gathered}
$$

We notice that the fourth-order phenomenological Eringen's model and the continualized nonlocal models are different for both the governing equations and the boundary conditions. Indeed, the dynamic term in Eq. (60) is twice less important than in Eq. (30). Furthermore, the governing differential equation of the Eringen's model is derivable from the principle of virtual work:

$$
\int_{0}^{L}\left\{M \delta\left(\frac{\mathrm{d}^{2} w}{\mathrm{~d} x^{2}}\right)-P \frac{\mathrm{d} w}{\mathrm{~d} x} \delta\left(\frac{\mathrm{d} w}{\mathrm{~d} x}\right)-m_{0} \omega^{2} w \delta(w)\right\} \mathrm{d} x
$$

The boundary conditions of the Eringen's model of this problem are

$$
\left[-\left\{\frac{\mathrm{d} M}{\mathrm{~d} x}+P \frac{\mathrm{d} w}{\mathrm{~d} x}\right\} \delta w\right]_{0}^{L}=0 ;\left[M \delta\left(\frac{\mathrm{d} w}{\mathrm{~d} x}\right)\right]_{0}^{L}=0
$$


The natural boundary conditions of the nonlocal Eringen's model Eq. (63) differ from the conditions obtained via the variationally based nonlocal approach Eqs. (60) and (61). The natural boundary conditions of the nonlocal Eringen's model can be expressed equivalently by:

$$
\begin{array}{r}
{\left[\left\{\left[E I-\left(e_{0} a\right)^{2} P\right] \frac{\mathrm{d}^{3} w}{\mathrm{~d} x^{3}}+\left[P+m_{0} \omega^{2}\left(e_{0} a\right)^{2}\right] \frac{\mathrm{d} w}{\mathrm{~d} x}\right\} \delta w\right]_{0}^{L}=0} \\
{\left[\left\{\left[E I-\left(e_{0} a\right)^{2} P\right] \frac{\mathrm{d}^{2} w}{\mathrm{~d} x^{2}}+m_{0} \omega^{2}\left(e_{0} a\right)^{2} w\right\} \delta\left(\frac{\mathrm{d} w}{\mathrm{~d} x}\right)\right]_{0}^{L}=0}
\end{array}
$$

In statics, Eqs. (64) and (65) coincide with Eqs. (60) and (61). In dynamics, the dynamic contribution in the boundary conditions differs between the phenomenological nonlocal Eringen's model and the conditions obtained through the variational approach Eqs. (60) and (61) (see also the analysis of Challamel et al. [47]).

The solution is provided for a simply supported beam. By substituting Eq. (32) into Eq. (56), we obtain

$$
m^{4}-\lambda\left[1+\left(\frac{e_{0} a m \pi}{L}\right)^{2}\right] m^{2}-\Omega^{2}\left[1+\left(\frac{e_{0} a m \pi}{L}\right)^{2}\right]=0
$$

In statics $(\omega=0)$, the nondimensional buckling load parameter is (see Fig. 2):

$$
\lambda=\min _{m} m^{2} \frac{1}{1+\left(\frac{e_{0} a m \pi}{L}\right)^{2}}=\frac{1}{1+\left(\frac{e_{0} a \pi}{L}\right)^{2}}
$$

The small length scale coefficient is obtained by equating Eq. (9) and Eq. (67):

$$
\frac{1}{1+\left(\frac{e_{0} a \pi}{L}\right)^{2}}=\left[\frac{2 n}{\pi} \sin \left(\frac{\pi}{2 n}\right)\right]^{2}
$$

In the sequel, the subscript $b$ denotes the buckling case (in statics). The exact buckling formula is expanded asymptotically as:

$$
1-\left(\frac{e_{0} \pi}{n}\right)^{2}+o\left(\frac{1}{n^{4}}\right)=1-\frac{1}{12}\left(\frac{\pi}{n}\right)^{2}+o\left(\frac{1}{n^{4}}\right)
$$

Thus, the best calibration of the length scale coefficient is

$$
e_{0, b}=\frac{1}{2 \sqrt{3}} \approx 0.288
$$

In free vibration $(P=0)$, the nondimensional frequency parameter is

$$
\Omega^{2}=m^{2} \frac{1}{1+\left(\frac{e_{0} a m \pi}{L}\right)^{2}}
$$

The variation of the nondimensional natural frequency parameter with respect to the number of elementary beam element is given in Fig. 3 for $m=1$. Again, the small length scale coefficient is obtained by comparing the solution of a microstructured beam and a nonlocal beam model. By doing so, we get

$$
m^{2} \frac{1}{1+\left(\frac{e_{0} a m \pi}{L}\right)^{2}}=\left[\frac{2 n}{\pi} \sin \left(\frac{\pi}{2 n}\right)\right]^{4}
$$

By expanding asymptotically for both sides of Eq. (72) for $m=1$, we obtain

$$
1-\left(\frac{e_{0} \pi}{n}\right)^{2}+o\left(\frac{1}{n^{4}}\right)=1-\frac{1}{6}\left(\frac{\pi}{n}\right)^{2}+o\left(\frac{1}{n^{4}}\right)
$$

In the sequel, we shall use the subscript $v$ to denote the free vibration case. The best calibration of the length scale coefficient is, for $m=1$,

$$
e_{0, v}=\frac{1}{\sqrt{6}} \approx 0.408
$$

This approach does not provide the same small length scale coefficient in buckling and in vibration: the small length scale coefficient is structural problem dependent. 


\subsection{Sixth-order phenomenological model}

A new attempt is made to obtain the sixth-order continualized equation through a phenomenological approach. Retaining the local equilibrium equation Eq. (4), a nonlocal constitutive law is postulated as follows:

$$
\left[1-\left(e_{0} a\right)^{2} \frac{\mathrm{d}^{2}}{\mathrm{~d} x^{2}}\right] M=E I\left[1+\left(e_{0} a\right)^{2} \frac{\mathrm{d}^{2}}{\mathrm{~d} x^{2}}\right] \frac{\mathrm{d}^{2}}{\mathrm{~d} x^{2}} w
$$

Multiplying Eq. (4) by $\left[1-\left(e_{0} a\right)^{2}\left(\mathrm{~d}^{2} / \mathrm{d} x^{2}\right)\right]$ and substituting Eq. (75) into Eq. (4), we obtain the governing differential equation

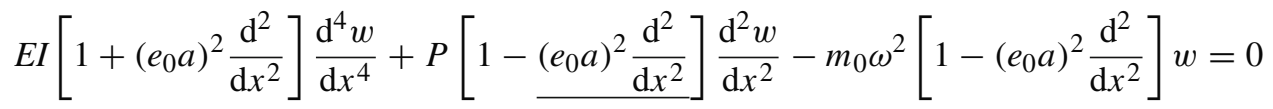

When compared to Eq. (40), it can be seen that Eq. (76) has an additional term that is underlined in the equation. This model is slightly different from a continualized nonlocal model. Furthermore, Eq. (76) may be obtained variationally by considering the energies $U, T$ and $W$ given by Eqs. (45), (29) and (46), respectively.

$$
\begin{aligned}
U(w) & =\int_{0}^{L} \frac{1}{2} E I\left[\left(\frac{\mathrm{d}^{2} w}{\mathrm{~d} x^{2}}\right)^{2}-\left(e_{0} a\right)^{2}\left(\frac{\mathrm{d}^{3} w}{\mathrm{~d} x^{3}}\right)^{2}\right] \mathrm{d} x \\
T(w) & =\int_{0}^{L} \frac{1}{2} m_{0} \omega^{2}\left[w^{2}+\left(e_{0} a\right)^{2}\left(\frac{\mathrm{d} w}{\mathrm{~d} x}\right)^{2}\right] \mathrm{d} x \\
W(w) & =\frac{1}{2} \int_{0}^{L} P\left[\left(\frac{\mathrm{d} w}{\mathrm{~d} x}\right)^{2}+\left(e_{0} a\right)^{2}\left(\frac{\mathrm{d}^{2} w}{\mathrm{~d} x^{2}}\right)^{2}\right] \mathrm{d} x
\end{aligned}
$$

The only difference between the two phenomenological models is the nonlocal term in the bending strain energy $U$. Thus, the application of the Hamilton principle leads to Eq. (76) with the natural boundary conditions

$$
\begin{gathered}
{\left[-\left\{E I\left(e_{0} a\right)^{2} \frac{\mathrm{d}^{5} w}{\mathrm{~d} x^{5}}+\left[E I-P\left(e_{0} a\right)^{2}\right] \frac{\mathrm{d}^{3} w}{\mathrm{~d} x^{3}}+\left[P+m_{0} \omega^{2}\left(e_{0} a\right)^{2}\right] \frac{\mathrm{d} w}{\mathrm{~d} x}\right\} \delta w\right]_{0}^{L}=0} \\
{\left[\left\{E I\left(e_{0} a\right)^{2} \frac{\mathrm{d}^{4} w}{\mathrm{~d} x^{4}}+\left[E I-P\left(e_{0} a\right)^{2}\right] \frac{\mathrm{d}^{2} w}{\mathrm{~d} x^{2}}\right\} \delta\left(\frac{\mathrm{d} w}{\mathrm{~d} x}\right)\right]_{0}^{L}=0} \\
{\left[\left\{E I\left(e_{0} a\right)^{2} \frac{\mathrm{d}^{3} w}{\mathrm{~d} x^{3}}\right\} \delta\left(\frac{\mathrm{d}^{2} w}{\mathrm{~d} x^{2}}\right)\right]_{0}^{L}=0}
\end{gathered}
$$

As explained in Sect. 2.3., the sixth-order space derivative (continualized and phenomenological) models introduce two additional boundary conditions, one at each end.

The solution is given by substituting Eq. (33) into Eq. (76):

$$
\left[1-\left(\frac{e_{0} a m \pi}{L}\right)^{2}\right] m^{4}-\lambda\left[1+\left(\frac{e_{0} a m \pi}{L}\right)^{2}\right] m^{2}-\bar{\Omega}^{2}\left[1+\left(\frac{e_{0} a m \pi}{L}\right)^{2}\right]=0
$$

Consider the static case $(\omega=0)$. Using Eqs. (83) and (90), the nondimensional buckling load parameter is given by (see Fig. 2):

$$
\lambda=\min _{m} m^{2} \frac{1-\left(\frac{e_{0} a m \pi}{L}\right)^{2}}{1+\left(\frac{e_{0} a m \pi}{L}\right)^{2}}
$$

As for the continualized models, it is observed from Fig. 2 that the fourth-order model provides more accurate results when compared to the sixth-order model. Moreover, the small length scale coefficient is obtained by equating Eqs. (9) and (84):

$$
m^{2} \frac{1-\left(\frac{e_{0} m \pi}{n}\right)^{2}}{1+\left(\frac{e_{0} m \pi}{n}\right)^{2}}=\left[\frac{2 n}{\pi} \sin \left(\frac{m \pi}{2 n}\right)\right]^{2}
$$


It yields the calibrated small length scale coefficient of

$$
e_{0, b}=\frac{1}{2 \sqrt{6}} \approx 0.204
$$

In the free vibration case $(P=0)$, the nondimensional frequency parameter is given by:

$$
\Omega^{2}=m^{2} \frac{1-\left(\frac{e_{0, v} m \pi}{n}\right)^{2}}{1+\left(\frac{e_{0, v} m \pi}{n}\right)^{2}}
$$

The variation of the nondimensional natural frequency parameter with respect to the number of elementary beam element is given in Fig. 3 for $m=1$. As for the fourth-order phenomenological model, equating the nondimensional frequency furnished by the discrete model with Eq. (10) and the frequency given in Eq. (87), we obtain the following calibrated small length scale coefficient:

$$
e_{0, v}=\frac{1}{2 \sqrt{3}} \approx 0.288
$$

The small length scale coefficient differs in statics and in buckling. This numerical result confirms that the nonlocal phenomenological model is structural problem dependent.

\subsection{General formulation of solutions}

It is worth noting that all the models may be presented in a unified way:

$$
E I\left[1+\gamma_{1}\left(e_{0} a\right)^{2} \frac{\mathrm{d}^{2}}{\mathrm{~d} x^{2}}\right] \frac{\mathrm{d}^{4} w}{\mathrm{~d} x^{4}}+P\left[1-\gamma_{2}\left(e_{0} a\right)^{2} \frac{\mathrm{d}^{2}}{\mathrm{~d} x^{2}}\right] \frac{\mathrm{d}^{2} w}{\mathrm{~d} x^{2}}-m_{0} \omega^{2}\left[1-\gamma_{3}\left(e_{0} a\right)^{2} \frac{\mathrm{d}^{2}}{\mathrm{~d} x^{2}}\right] w=0
$$

where three control scalar parameters, namely $\gamma_{1}, \gamma_{2}, \gamma_{3}$ are introduced with $\left(\gamma_{1}, \gamma_{2}, \gamma_{3}\right)$ is equal to $(0,1,2),(1,0,1),(0,1,1)$ and $(1,1,1)$ for the fourth-order continualized model, its equivalent sixth-order derivative counterpart, the phenomenological fourth-order derivative and the phenomenological sixth-order derivative, respectively. In the continualized models, the small length scale coefficient is structural problem independent and is equal to $1 / \sqrt{12}$. In the phenomenological models, the value of this coefficient is unknown, and it has to be calibrated based on the asymptotic equivalence between the continuous and the discrete models. Thus, all previous results are obtained in a general form by using those parameters $\gamma_{1}, \gamma_{2}, \gamma_{3}$ and $e_{0}$ equal to $1 / \sqrt{12}$ for the continualized models.

The substitution of Eq. (32) into Eq. (89) furnishes

$$
\left[1-\gamma_{1}\left(\frac{e_{0} a m \pi}{L}\right)^{2}\right] m^{4}-\lambda\left[1+\gamma_{2}\left(\frac{e_{0} a m \pi}{L}\right)^{2}\right] m^{2}-\bar{\Omega}^{2}\left[1+\gamma_{3}\left(\frac{e_{0} a m \pi}{L}\right)^{2}\right]=0
$$

By using Eq. (90), the nondimensional buckling load parameter $(\omega=0)$, is (see Fig. 2):

$$
\lambda=\min _{m} m^{2} \frac{1-\gamma_{1}\left(\frac{e_{0} a m \pi}{L}\right)^{2}}{1+\gamma_{2}\left(\frac{e_{0} a m \pi}{L}\right)^{2}}
$$

It is observed from Fig. 2 and explained above that the fourth-order models provide more accurate results when compared to the sixth-order models (continualized and phenomenological). The small length scale coefficient is calibrated by comparing Eq. (9) and Eq. (91):

$$
m^{2} \frac{1-\gamma_{1}\left(\frac{e_{0} m \pi}{n}\right)^{2}}{1+\gamma_{2}\left(\frac{e_{0} m \pi}{n}\right)^{2}}=\left[\frac{2 n}{\pi} \sin \left(\frac{m \pi}{2 n}\right)\right]^{2}
$$

Thus, the general expression of the best calibration of the length scale coefficient is

$$
e_{0, b}=\frac{1}{\sqrt{\gamma_{1}+\gamma_{2}}} \frac{1}{2 \sqrt{3}}
$$


It is observed that the small length scale coefficient depends on the model. Thus, it is equal to 0.144 with the phenomenological sixth-order space derivative model and 0.288 for the other three models. is:

In a similar manner, in the free vibration case $(P=0)$, the natural nondimensional frequency parameter

$$
\Omega^{2}=m^{2} \frac{1-\gamma_{1}\left(\frac{e_{0} a m \pi}{L}\right)^{2}}{1+\gamma_{3}\left(\frac{e_{0} a m \pi}{L}\right)^{2}}
$$

As for the buckling load, the small length scale coefficient is obtained by comparing the solution of a microstructured beam (see Sect. 2.1.) and a nonlocal beam model. In view of Eqs. (10) and (94), we can write:

$$
m^{2} \frac{1-\gamma_{1}\left(e_{0, v} n m \pi\right)^{2}}{1+\gamma_{3}\left(e_{0, v} n m \pi\right)^{2}}=\left[\frac{2 n}{\pi} \sin \left(\frac{m \pi}{2 n}\right)\right]^{4}
$$

Thus, the best calibration of the length scale coefficient is, for $m=1$,

$$
e_{0, v}=\frac{1}{\sqrt{\gamma_{1}+\gamma_{3}}} \frac{1}{\sqrt{6}}
$$

The small length scale coefficient depends on the model but not in the same manner than in statics. Indeed, the parameter $\gamma_{2}$ has been replaced by $\gamma_{3}$. By definition, for the continualized models, the small length scale coefficient is structural problem independent and it is equal to $1 / \sqrt{12}$. In the phenomenological approaches, the calibration of the coefficient shows that it is structural problem dependent. Thus, these approaches are less robust than the continualized models.

\section{Extension to plate}

The previous derivation will now be extended to plates. In a recent article, Challamel et al. [20] developed a continualized plate model based on Padé approximant and a model called hybrid continualized model. In contrast to the existing nonlocal plate models governed by a fourth-order differential equation, the hybrid continualized model introduced a new sixth-order derivative in the equation. The solution requires to two additional boundary conditions and this new constrain raises the validity issue of such a model. We have shown that for a beam, it is possible to build continualized models leading to sixth-order derivative terms in the governing differential equation. In this part, we briefly derive the governing differential equations obtained with the nonlocal Eringen plate theory (widely investigated in the literature) and continualized models [20].

\subsection{Microstructured beam-grid model}

As for a beam, the lattice model is considered as the reference model for calibration of the small length scale coefficient at a later stage. Following the idea presented in Sect. 2.1., a microstructured beam-grid for modeling can be reproduced by an assembly of chain net systems of rigid straight elements connected at frictionless joints where rotations are localized as shown in Fig. 3. Bending deformation of the beam-grid is made possible by rotational springs at individual nodes. The torsion deformation is modeled by a rectangular repetitive unit cell composed of four rigid beam elements with springs in the central domain [34]. The masses are lumped at the nodes of the beam-grid.

So, consider a vibrating microstructured beam-grid model under an initial biaxial loads $P$ and $s P$ as shown in Figs. 3 and 4. Although it is the same notation than in the beam case, hereinafter, $P$ denotes a load per unit length. The particular cases of $s=0$ (uniaxial load) and $s=1$ (load is the same along both directions) have been studied by Zhang et al. [48] and Zhang et al. [18], respectively. In the lattice plate model, the moment-curvature relations are given by:

$$
\begin{aligned}
& M_{x}^{i, j}=D\left(\frac{w_{i+1, j}-2 w_{i, j}+w_{i-1, j}}{\Delta x^{2}}+v \frac{w_{i, j+1}-2 w_{i, j}+w_{i, j-1}}{\Delta y^{2}}\right) \\
& M_{y}^{i, j}=D\left(\frac{w_{i, j+1}-2 w_{i, j}+w_{i, j-1}}{\Delta y^{2}}+v \frac{w_{i+1, j}-2 w_{i, j}+w_{i-1, j}}{\Delta x^{2}}\right) \\
& M_{x y}^{i, j}=M_{y x}^{i, j}=D(1-v) \frac{-w_{i, j+1}+w_{i+1, j+1}+w_{i, j}-w_{i+1, j}}{\Delta x \Delta y}
\end{aligned}
$$



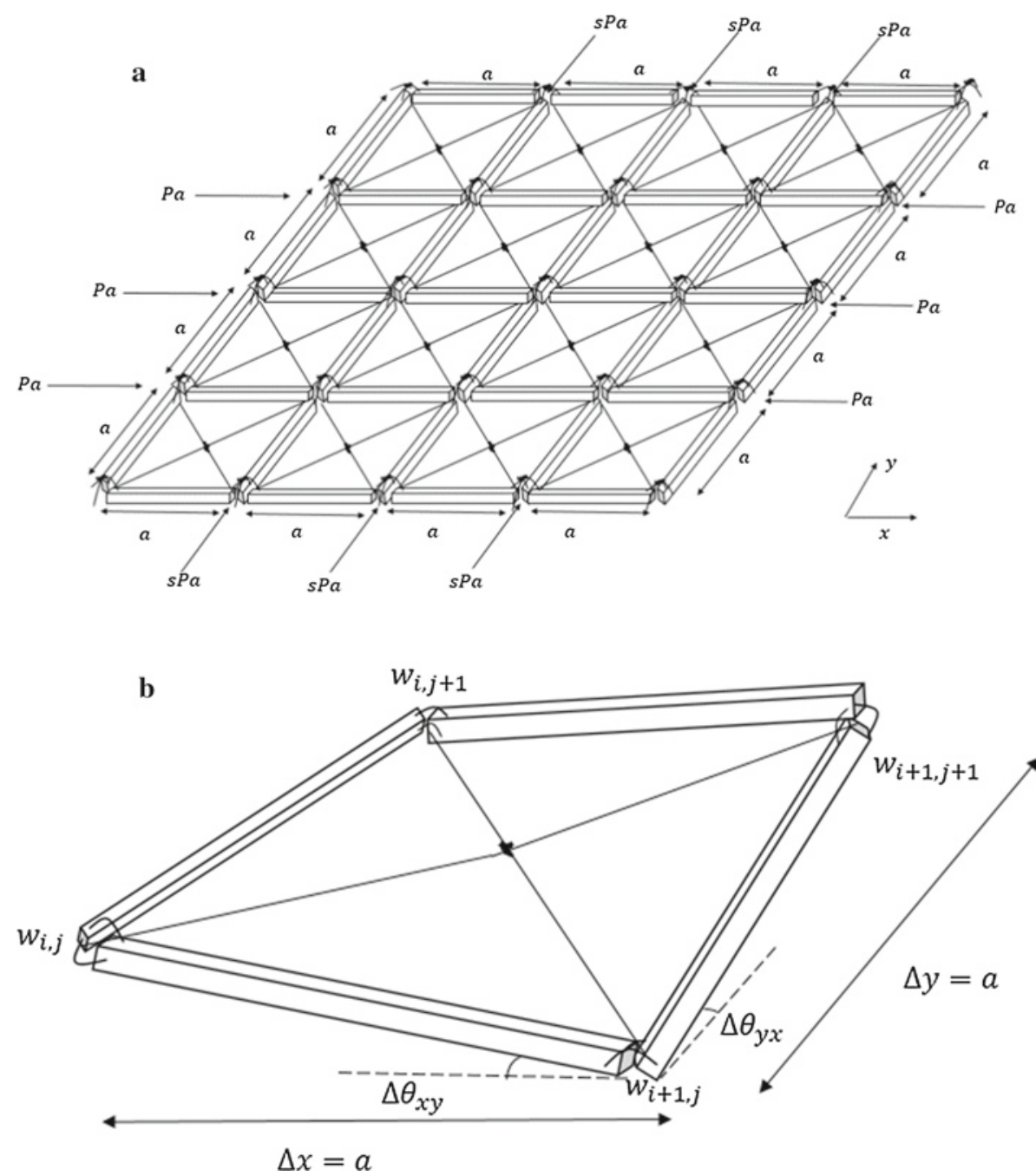

Fig. 4 a Microstructured beam-grid model. b Torsional deformation of a unit cell in a microstructured beam-grid model

and the discrete equation of motion by

$$
\begin{aligned}
& \frac{M_{x x}^{i+1, j}-2 M_{x x}^{i, j}+M_{x x}^{i-1, j}}{\Delta x^{2}}+2 \frac{M_{x y}^{i, j}-M_{x y}^{i-1, j}-M_{x y}^{i, j-1}+M_{x y}^{i-1, j-1}}{\Delta x \Delta y}+\frac{M_{y y}^{i, j+1}-2 M_{y y}^{i, j}+M_{y y}^{i, j-1}}{\Delta y^{2}} \\
& +P\left(\frac{w_{i+1, j}-2 w_{i, j}+w_{i-1, j}}{\Delta x^{2}}+s \frac{w_{i, j+1}-2 w_{i, j}+w_{i, j-1}}{\Delta y^{2}}\right)-m_{0} \omega^{2} w_{i, j}=0
\end{aligned}
$$

where $w_{i, j}, M_{x x}, M_{y y}, M_{x y}$ are the displacement, the bending moments and the twisting moment at node $(i, j)$, respectively. $\Delta x$ and $\Delta y$ are the lengths of discrete rigid elements in the $x$-and $y$-directions, given, employing a square lattice for an isotropic material, by $\Delta x=\Delta y=a=\alpha L / n_{y}=L / n_{x}$, with $n_{x}$ and $n_{y}$ are the numbers of discrete rigid elements in the $x$ - and $y$-directions, also seen, as for a beam, as the ratio between the length of the plate and the interatomic length.

Equations (97)-(100) are the centered finite schemes of the local moment-curvature relations and the local equation of motion for a plate, following the Kirchhoff plate model [49] 


$$
\begin{gathered}
M_{x x}=D\left(\frac{\partial^{2}}{\partial x^{2}} w+v \frac{\partial^{2}}{\partial y^{2}} w\right) \\
M_{y y}=D\left(\frac{\partial^{2} w}{\partial y^{2}}+v \frac{\partial^{2}}{\partial x^{2}} w\right) \\
M_{x y}=D(1-v) \frac{\partial^{2}}{\partial x \partial y} w \\
\frac{\partial^{2} M_{x x}}{\partial x^{2}}+2 \frac{\partial^{2} M_{x y}}{\partial x \partial y}+\frac{\partial^{2} M_{y y}}{\partial y^{2}}+P\left(\frac{\partial^{2} w}{\partial x^{2}}+s \frac{\partial^{2} w}{\partial y^{2}}\right)-m_{0} \omega^{2} w=0
\end{gathered}
$$

where $M_{x x}, M_{y y}$ are the bending moments, $M_{x y}$ the twisting moment, $D$ the flexural rigidity of the continuous plate, and $v$ the Poisson ratio.

In view of Eqs. (97)-(99), and Eq. (100) gives the linear difference equation of the plate lattice problem

$$
\alpha^{2} n_{y}^{4} L^{i, j}+n_{y}^{2} \alpha^{2} \lambda_{\text {(micro) }}\left(H_{x}^{i, j}+s H_{y}^{i, j}\right)-\alpha^{2} \Omega_{(\text {micro })}^{2} w_{i, j}=0
$$

where

$$
\begin{aligned}
L^{i, j}= & 20 w_{i, j}+\left(w_{i, j-2}+w_{i, j+2}+w_{i-2, j}+w_{i+2, j}\right)-8\left(w_{i, j-1}+w_{i, j+1}+w_{i-1, j}+w_{i+1, j}\right) \\
& +2\left(w_{i+1, j-1}+w_{i+1, j+1}+w_{i-1, j-1}+w_{i-1, j+1}\right) \\
H_{y}^{i, j}= & w_{i, j-1}-2 w_{i, j}+w_{i, j+1} \\
H_{x}^{i, j}= & w_{i-1, j}-2 w_{i, j}+w_{i+1, j} \\
\Omega_{\text {(micro) }}^{2}= & \frac{m_{0} \omega^{2}(\alpha L)^{4}}{D} ; \lambda_{\text {(micro) }}=\frac{P(\alpha L)^{2}}{D}
\end{aligned}
$$

where $\Omega_{\text {(micro) }}$ is the dimensionless vibration frequency parameter of the microstructured beam-grid model and $\lambda$ the nondimensional buckling stress parameter. Equation (105) may also be derived by using the Hamilton principle [20].

Equation (105) is the finite difference scheme of the local continuous governing differential equation [20]:

$$
D\left[\frac{\partial^{4} w}{\partial x^{4}}+2 \frac{\partial^{4} w}{\partial x^{2} \partial y^{2}}+\frac{\partial^{4} w}{\partial y^{4}}\right]-m_{0} \omega^{2} w+P\left(\frac{\partial^{2} w}{\partial x^{2}}+s \frac{\partial^{2} w}{\partial y^{2}}\right)=0
$$

Consider now a microstructured beam-grid model with simply supported boundary conditions. The discrete displacement can be expressed as a double Fourier sine series as:

$$
w_{i, j}=w_{0} \sin \frac{m \pi i}{n_{x}} \sin \frac{n \pi j}{n_{y}}=w_{0} \sin \frac{\alpha m \pi i}{n_{y}} \sin \frac{n \pi j}{n_{y}}
$$

By substituting Eq. (111) into Eq. (105), one obtains the vibration frequency parameter in the general case:

$$
\Omega_{\text {(micro) }}^{2}=4 n_{y}^{4}\left(2-\cos \frac{m \pi}{n_{x}}+\cos \frac{n \pi}{n_{y}}\right)^{2}-2 \lambda_{(\text {micro })} n_{y}^{2}\left(1+s-\cos \frac{m \pi}{n_{x}}-s \cos \frac{n \pi}{n_{y}}\right)
$$

This equation coincides with the one developed by Challamel et al. [20]. The vibration frequency remains positive as long as the load is lower than the buckling load. Likewise, the critical buckling load parameter is given by

$$
\lambda_{\text {(micro) }}=\min _{(m, n)}\left(-\frac{2 n_{y}^{2}\left(\cos \frac{m \pi}{n_{x}}+\cos \frac{n \pi}{n_{y}}-2\right)^{2}}{\left(\cos \frac{m \pi}{n_{x}}+s \cos \frac{n \pi}{n_{y}}-1-s\right)}\right)
$$

Assuming that the loads are the same along the two directions $x$ and $y$, i.e. $s=1$, Eq. (112) becomes:

$$
\Omega_{\text {(micro) }}^{2}=2 n_{y}^{2}\left(2-\cos \frac{m \pi}{n_{x}}-\cos \frac{n \pi}{n_{y}}\right)\left[2 n_{y}^{2}\left(2-\cos \frac{m \pi}{n_{x}}-\cos \frac{n \pi}{n_{y}}\right)-\lambda_{\text {(micro) }}\right]
$$


Remarkably, Zhang et al. [18] derived the same result. Moreover, assuming a uniaxial load, $s=0$, the squared vibration frequency parameter becomes:

$$
\Omega_{\text {(micro) }}^{2}=4 n_{y}^{4}\left(2-\cos \frac{\alpha m \pi}{n_{y}}-\cos \frac{n \pi}{n_{y}}\right)^{2}-2 \lambda_{(\text {micro })} n_{y}^{2}\left(1-\cos \frac{\alpha m \pi}{n_{y}}\right)
$$

In this case, the minimum of $\lambda_{\text {(micro) }}$, depends on $\alpha$ and $n_{y}$, occurs when $n=m=1$ and the critical buckling load results in

$$
\lambda_{(\text {micro })}=\frac{2 n_{y}^{2}\left(\cos \frac{\alpha \pi}{n_{y}}+\cos \frac{\pi}{n_{y}}-2\right)^{2}}{1-\cos \frac{\alpha \pi}{n_{y}}}
$$

Now we present the nonlocal Eringen's model and different continualized models. This lattice model is the reference model for the calibration of the small length scale coefficient. Thus, the aim is to build a nonlocal continuous model such that the results match with those of the microstructured beam-grid model. As in the beam problem, we consider two kinds of models. The first kind is based on the equivalence between the continuous and discrete models through the continualization of finite equations (presented above). The second kind of models is based on the use of postulated nonlocal constitutive laws and equilibrium equations; the most famous of them being the nonlocal Eringen's model.

\subsection{Fourth-order continualized model}

Recently, Challamel et al. [20] proposed a continualized model based on Padé approximant. As in the beam problem, we assume a dense lattice. By using the Padé approximants as presented in Sect. 2.3. and extending to two dimensions, we define the following two-dimensional operators at node $(i, j)$

$$
\begin{aligned}
& L_{a, x}=\left.\frac{\partial^{2} w}{\partial x^{2}}\right|_{i, j}=\left(\frac{\partial}{\partial x}\right)_{i, j}^{2} \\
& L_{a, y}=\left.\frac{\partial^{2} w}{\partial y^{2}}\right|_{i, j}=\left(\frac{\partial}{\partial y}\right)_{i, j}^{2}
\end{aligned}
$$

According to Eq. (15), the continualized approximation of this operator is provided by the following expression

$$
\begin{aligned}
& L_{a, x}=\frac{4}{a^{2}} \sinh ^{2}\left(\frac{a}{2} \frac{\partial}{\partial x}\right)=\frac{\frac{\partial^{2}}{\partial x^{2}}}{1-\frac{a^{2}}{12} \frac{\partial^{2}}{\partial x^{2}}}+o\left(a^{2}\right) \\
& L_{a, y}=\frac{4}{a^{2}} \sinh ^{2}\left(\frac{a}{2} \frac{\partial}{\partial y}\right)=\frac{\frac{\partial^{2}}{\partial y^{2}}}{1-\frac{a^{2}}{12} \frac{\partial^{2}}{\partial y^{2}}}+o\left(a^{2}\right)
\end{aligned}
$$

Following the study of Rosenau [25]

$$
\begin{aligned}
& L_{a, x}^{\frac{1}{2}}=\frac{\frac{\partial}{\partial x}}{1-\frac{a^{2}}{24} \frac{\partial^{2}}{\partial x^{2}}}+o\left(a^{2}\right) \\
& L_{a, y}^{\frac{1}{2}}=\frac{\frac{\partial}{\partial y}}{1-\frac{a^{2}}{24} \frac{\partial^{2}}{\partial y^{2}}}+o\left(a^{2}\right)
\end{aligned}
$$

By applying Eqs. (118) and (119) on Eqs. (97)-(99), we obtain [20] 


$$
\begin{aligned}
& M_{x}=D\left(L_{a, x}+v L_{a, y}\right) w=D\left(\frac{\frac{\partial^{2}}{\partial x^{2}}}{1-\frac{a^{2}}{12} \frac{\partial^{2}}{\partial x^{2}}} w+v \frac{\frac{\partial^{2}}{\partial y^{2}}}{1-\frac{a^{2}}{12} \frac{\partial^{2}}{\partial y^{2}}} w\right)+o\left(a^{2}\right) \\
& M_{y}=D\left(L_{a, y}+v L_{a, x}\right) w=D\left(\frac{\frac{\partial^{2}}{\partial y^{2}}}{1-\frac{a^{2}}{12} \frac{\partial^{2}}{\partial y^{2}}} w+v \frac{\frac{\partial^{2}}{\partial x^{2}}}{1-\frac{a^{2}}{12} \frac{\partial^{2}}{\partial x^{2}}} w\right)+o\left(a^{2}\right) \\
& M_{x y}=D\left(L_{a, x} L_{a, y}\right)^{1 / 2} w=D(1-v) \frac{\frac{\partial^{2}}{\partial x \partial y}}{\left(1-\frac{a^{2}}{24} \frac{\partial^{2}}{\partial x^{2}}\right)\left(1-\frac{a^{2}}{24} \frac{\partial^{2}}{\partial y^{2}}\right)} w+o\left(a^{2}\right) \\
& L_{a, x} M_{x x}+2\left(L_{a, x} L_{a, y}\right)^{1 / 2} M_{x y}+L_{a, y} M_{y y}+P L_{a, x} w+s P L_{a, y} w-m_{0} \omega^{2} w \\
& =\frac{\frac{\partial^{2}}{\partial x^{2}}}{1-\frac{a^{2}}{12} \frac{\partial^{2}}{\partial x^{2}}} M_{x x}+2 \frac{\frac{\partial^{2}}{\partial x \partial y}}{\left[1-\frac{a^{2}}{24} \frac{\partial^{2}}{\partial x^{2}}\right]\left[1-\frac{a^{2}}{24} \frac{\partial^{2}}{\partial y^{2}}\right]} M_{x y}+\frac{\frac{\partial^{2}}{\partial y^{2}}}{1-\frac{a^{2}}{12} \frac{\partial^{2}}{\partial y^{2}}} M_{y y} \\
& +P \frac{\frac{\partial^{2}}{\partial x^{2}}}{1-\frac{a^{2}}{12} \frac{\partial^{2}}{\partial x^{2}}} w+s P \frac{\frac{\partial^{2}}{\partial y^{2}}}{1-\frac{a^{2}}{12} \frac{\partial^{2}}{\partial y^{2}}} w-m_{0} \omega^{2} w+o\left(a^{2}\right)=0
\end{aligned}
$$

By using the Taylor expansion, Eqs. (120)-(122) lead to a mixed gradient-nonlocal law, given by:

$$
\begin{aligned}
M_{x} & =D\left(\frac{\partial^{2}}{\partial x^{2}}\left(1+\frac{a^{2}}{12} \frac{\partial^{2}}{\partial x^{2}}\right) w+v \frac{\partial^{2}}{\partial y^{2}}\left(1+\frac{a^{2}}{12} \frac{\partial^{2}}{\partial y^{2}}\right) w\right)+o\left(a^{2}\right) \\
M_{y} & =D\left(\frac{\partial^{2}}{\partial y^{2}}\left(1+\frac{a^{2}}{12} \frac{\partial^{2}}{\partial y^{2}}\right) w+v \frac{\partial^{2}}{\partial x^{2}}\left(1+\frac{a^{2}}{12} \frac{\partial^{2}}{\partial x^{2}}\right) w\right)+o\left(a^{2}\right) \\
M_{x y} & =D(1-v)\left(1+\frac{a^{2}}{24} \frac{\partial^{2}}{\partial x^{2}}\right)\left(1+\frac{a^{2}}{24} \frac{\partial^{2}}{\partial y^{2}}\right) \frac{\partial^{2}}{\partial x \partial y} w+o\left(a^{2}\right)
\end{aligned}
$$

Multiplying Eq. (123) by $\left[1-\left(a^{2} / 12\right) \partial^{2} / \partial x^{2}\right]^{2}\left[1+\left(e_{0} a\right)^{2} \partial^{2} / \partial y^{2}\right]^{2}$ and using the Taylor expansion at the second order in $a$, we obtain

$$
\begin{aligned}
& D\left[\frac{\partial^{4} w}{\partial x^{4}}+2 \frac{\partial^{4} w}{\partial x^{2} \partial y^{2}}+\frac{\partial^{4} w}{\partial y^{4}}-\frac{a^{2}}{3}\left(\frac{\partial^{6} w}{\partial x^{4} \partial y^{2}}+\frac{\partial^{6} w}{\partial x^{2} \partial y^{4}}\right)\right. \\
& +\frac{a^{4}}{96}\left(\frac{v-3}{3} \frac{\partial^{8} w}{\partial x^{6} \partial y^{2}}+\frac{v-3}{3} \frac{\partial^{8} w}{\partial x^{2} \partial y^{6}}+\frac{15 v-7}{3} \frac{\partial^{8} w}{\partial x^{4} \partial y^{4}}-\frac{2}{3} \frac{\partial^{8} w}{\partial x^{8}}-\frac{2}{3} \frac{\partial^{8} w}{\partial y^{8}}\right) \\
& +\frac{a^{6}}{576}\left(\frac{\partial^{10} w}{\partial x^{2} \partial y^{8}}+\frac{\partial^{10} w}{\partial x^{8} \partial y^{2}}+\frac{48-13 v}{12} \frac{\partial^{10} w}{\partial x^{4} \partial y^{6}}+\frac{48-13 v}{12} \frac{\partial^{10} w}{\partial x^{6} \partial y^{4}}\right) \\
& +\frac{a^{8}}{4608}\left(\frac{5-9 v}{9} \frac{\partial^{10} w}{\partial x^{4} \partial y^{8}}+\frac{5-9 v}{9} \frac{\partial^{10} w}{\partial x^{8} \partial y^{4}}+\frac{13-13 v}{9} \frac{\partial^{10} w}{\partial x^{6} \partial y^{6}}\right. \\
& \left.+\frac{1-v}{9} \frac{\partial^{10} w}{\partial x^{10} \partial y^{2}}+\frac{1-v}{9} \frac{\partial^{10} w}{\partial x^{2} \partial y^{10}}+\frac{48-13 v}{12} \frac{\partial^{10} w}{\partial x^{6} \partial y^{4}}\right) \\
& +\frac{a^{10}(1-v)}{995328}\left(2 \frac{\partial^{10} w}{\partial x^{6} \partial y^{8}}+2 \frac{\partial^{10} w}{\partial x^{8} \partial y^{6}}+\frac{\partial^{10} w}{\partial x^{10} \partial y^{4}}+\frac{\partial^{10} w}{\partial x^{4} \partial y^{10}}\right) \\
& \left.+\frac{a^{12}(v-1)}{11943936}\left(\frac{\partial^{10} w}{\partial x^{10} \partial y^{6}}+\frac{\partial^{10} w}{\partial x^{6} \partial y^{10}}+2 \frac{\partial^{10} w}{\partial x^{8} \partial y^{8}}\right)\right] \\
& +p\left[\frac{\partial^{2} w}{\partial x^{2}}+s \frac{\partial^{2} w}{\partial y^{2}}-\frac{a^{2}}{12}\left(2(s+1) \frac{\partial^{4} w}{\partial x^{2} \partial y^{2}}+\frac{\partial^{4} w}{\partial x^{4}}+s \frac{\partial^{4} w}{\partial y^{4}}\right)\right. \\
& +\frac{a^{4}}{144}\left(\frac{\partial^{6} w}{\partial x^{6}}+(s+1) \frac{\partial^{6} w}{\partial x^{4} \partial y^{2}}+(s+1) \frac{\partial^{6} w}{\partial x^{2} \partial y^{4}}+s \frac{\partial^{6} w}{\partial y^{6}}\right)-\frac{a^{6}}{1728}\left(\frac{\partial^{8} w}{\partial x^{8}}+s \frac{\partial^{8} w}{\partial y^{8}}\right)
\end{aligned}
$$




$$
\begin{aligned}
& \left.-\frac{a^{8}(s+1)}{20736}\left(\frac{\partial^{10} w}{\partial x^{8} \partial y^{2}}+\frac{\partial^{10} w}{\partial x^{2} \partial y^{8}}\right)\right] \\
& -m_{0} \omega^{2}\left[1-\frac{a^{2}}{6}\left(\frac{\partial^{2} w}{\partial x^{2}}+\frac{\partial^{2} w}{\partial y^{2}}\right)+\frac{a^{4}}{48}\left(\frac{\partial^{4} w}{\partial x^{4}}+\frac{5}{3} \frac{\partial^{4} w}{\partial x^{2} \partial y^{2}}+\frac{\partial^{4} w}{\partial y^{4}}\right)\right. \\
& \left.-\frac{a^{6}}{1728}\left(\frac{\partial^{6} w}{\partial x^{6}}+\frac{\partial^{6} w}{\partial y^{6}}+\frac{\partial^{6} w}{\partial x^{4} \partial y^{2}}+\frac{\partial^{6} w}{\partial x^{2} \partial y^{4}}\right)-\frac{a^{8}}{20736}\left(\frac{\partial^{8} w}{\partial x^{6} \partial y^{2}}+\frac{\partial^{8} w}{\partial x^{4} \partial y^{4}}+\frac{\partial^{8} w}{\partial x^{2} \partial y^{6}}\right)\right] \\
& +o\left(a^{12}\right)=0
\end{aligned}
$$

As in the continualized beam models, we define a small length scale coefficient, structural independent, $e_{0}$ equal to $1 / \sqrt{12}$. This coefficient is constant and structural problem independent. Neglecting the higher-order terms in $a$, one obtains the approximation:

$$
\begin{aligned}
& D\left[\frac{\partial^{4} w}{\partial x^{4}}+2 \frac{\partial^{4} w}{\partial x^{2} \partial y^{2}}+\frac{\partial^{4} w}{\partial y^{4}}-\frac{a^{2}}{3}\left(\frac{\partial^{6} w}{\partial x^{4} \partial y^{2}}+\frac{\partial^{6} w}{\partial x^{2} \partial y^{4}}\right)\right] \\
& +P\left[\frac{\partial^{2} w}{\partial x^{2}}+s \frac{\partial^{2} w}{\partial y^{2}}-\frac{a^{2}}{12}\left(2(s+1) \frac{\partial^{4} w}{\partial x^{2} \partial y^{2}}+\frac{\partial^{4} w}{\partial x^{4}}+s \frac{\partial^{4} w}{\partial y^{4}}\right)\right] \\
& -m_{0} \omega^{2}\left[1-\frac{a^{2}}{6}\left(\frac{\partial^{2}}{\partial x^{2}}+\frac{\partial^{2}}{\partial y^{2}}\right)\right] w+o\left(a^{2}\right)=0
\end{aligned}
$$

Equation (128) can be directly derived from the continualization of the discrete governing differential equation Eq. (105). Using the pseudodifferential operator defined in Eqs. (118) and (119):

$$
\begin{aligned}
& \frac{16}{a^{4}} D\left[\sinh ^{4}\left(\frac{a}{2} \frac{\partial}{\partial x}\right)+2 \sinh ^{2}\left(\frac{a}{2} \frac{\partial}{\partial x}\right) \sinh ^{2}\left(\frac{a}{2} \frac{\partial}{\partial y}\right)+\sinh ^{4}\left(\frac{a}{2} \frac{\partial}{\partial y}\right)\right] w-m_{0} \omega^{2} w \\
& +P \frac{4}{a^{2}}\left[\sinh ^{2}\left(\frac{a}{2} \frac{\partial}{\partial x}\right)+s \sinh ^{2}\left(\frac{a}{2} \frac{\partial}{\partial y}\right)\right] w=0
\end{aligned}
$$

which can be approximated by:

$$
\begin{aligned}
& D\left[\frac{\frac{\partial^{4} w}{\partial x^{4}}}{\left(1-\frac{a^{2}}{12} \frac{\partial^{2}}{\partial x^{2}}\right)^{2}}+2 \frac{\frac{\partial^{4} w}{\partial x^{2} \partial y^{2}}}{\left(1-\frac{a^{2}}{12} \frac{\partial^{2}}{\partial x^{2}}\right)\left(1-\frac{a^{2}}{12} \frac{\partial^{2}}{\partial y^{2}}\right)}+\frac{\frac{\partial^{4} w}{\partial y^{4}}}{\left(1-\frac{a^{2}}{12} \frac{\partial^{2}}{\partial y^{2}}\right)^{2}}\right]-m_{0} \omega^{2} w \\
& +P\left(\frac{\frac{\partial^{2} w}{\partial x^{2}}}{1-\frac{a^{2}}{12} \frac{\partial^{2}}{\partial x^{2}}}+s \frac{\frac{\partial^{2} w}{\partial y^{2}}}{1-\frac{a^{2}}{12} \frac{\partial^{2}}{\partial y^{2}}}\right)+o\left(a^{2}\right)=0
\end{aligned}
$$

and we multiply by $\left[1-(a / 12)^{2}\left(\partial^{2} / \partial x^{2}\right)\right]^{2}\left[1-(a / 12)^{2}\left(\partial^{2} / \partial y^{2}\right)\right]^{2}$

$$
\begin{aligned}
& D\left[\left(1-\frac{a^{2}}{12} \frac{\partial^{2}}{\partial y^{2}}\right)^{2} \frac{\partial^{4} w}{\partial x^{4}}+2\left(1-\frac{a^{2}}{12} \frac{\partial^{2}}{\partial x^{2}}\right)\left(1-\frac{a^{2}}{12} \frac{\partial^{2}}{\partial y^{2}}\right) \frac{\partial^{4} w}{\partial x^{2} \partial y^{2}}+\left(1-\frac{a^{2}}{12} \frac{\partial^{2}}{\partial x^{2}}\right)^{2} \frac{\partial^{4} w}{\partial y^{4}}\right] \\
& -m_{0} \omega^{2} w+P\left(\frac{\partial^{2} w}{\partial x^{2}}+s \frac{\partial^{2} w}{\partial y^{2}}\right)-\frac{a^{2}}{6} P(s+1) \frac{\partial^{4} w}{\partial x^{2} \partial y^{2}}-\frac{a^{2}}{12} P\left(\frac{\partial^{4} w}{\partial x^{4}}+s \frac{\partial^{4} w}{\partial y^{4}}\right) \\
& +\frac{a^{4}}{144} P\left(\frac{\partial^{6} w}{\partial x^{6}}+2 \frac{\partial^{6} w}{\partial x^{4} \partial y^{2}}+s \frac{\partial^{6} w}{\partial y^{6}}+2 s \frac{\partial^{6} w}{\partial x^{2} \partial y^{4}}\right)-\frac{a^{6}}{1728} P(s+1) \frac{\partial^{8} w}{\partial x^{4} \partial y^{4}}+o\left(a^{6}\right)=0
\end{aligned}
$$

Or

$$
\begin{aligned}
& D\left[\frac{\partial^{4} w}{\partial x^{4}}+2 \frac{\partial^{4} w}{\partial x^{2} \partial y^{2}}+\frac{\partial^{4} w}{\partial y^{4}}-\frac{a^{2}}{3}\left(\frac{\partial^{6} w}{\partial x^{4} \partial y^{2}}+\frac{\partial^{6} w}{\partial x^{2} \partial y^{4}}\right)+\frac{a^{4}}{36} \frac{\partial^{8} w}{\partial x^{4} \partial y^{4}}\right] \\
& -m_{0} \omega^{2} w+\frac{a^{2}}{6} m_{0} \omega^{2}\left(\frac{\partial^{2} w}{\partial x^{2}}+\frac{\partial^{2} w}{\partial y^{2}}\right)
\end{aligned}
$$




$$
\begin{aligned}
& +P\left(\frac{\partial^{2} w}{\partial x^{2}}+s \frac{\partial^{2} w}{\partial y^{2}}\right)-\frac{a^{2}}{6} P(s+1) \frac{\partial^{4} w}{\partial x^{2} \partial y^{2}}-\frac{a^{2}}{12} P\left(\frac{\partial^{4} w}{\partial x^{4}}+s \frac{\partial^{4} w}{\partial y^{4}}\right) \\
& +\frac{a^{4}}{144} P\left(\frac{\partial^{6} w}{\partial x^{6}}+2 \frac{\partial^{6} w}{\partial x^{4} \partial y^{2}}+s \frac{\partial^{6} w}{\partial y^{6}}+2 s \frac{\partial^{6} w}{\partial x^{2} \partial y^{4}}\right)-\frac{a^{6}}{1728} P(s+1) \frac{\partial^{8} w}{\partial x^{4} \partial y^{4}}+o\left(a^{6}\right)=0
\end{aligned}
$$

Neglecting the higher-order term in $a$ in Eq. (132) yields Eq. (128):

$$
\begin{aligned}
& D\left[\frac{\partial^{4} w}{\partial x^{4}}+2 \frac{\partial^{4} w}{\partial x^{2} \partial y^{2}}+\frac{\partial^{4} w}{\partial y^{4}}-\frac{a^{2}}{3}\left(\frac{\partial^{6} w}{\partial x^{4} \partial y^{2}}+\frac{\partial^{6} w}{\partial x^{2} \partial y^{4}}\right)\right]-m_{0} \omega^{2} w \\
& +\frac{a^{2}}{6} m_{0} \omega^{2}\left(\frac{\partial^{2} w}{\partial x^{2}}+\frac{\partial^{2} w}{\partial y^{2}}\right)+P\left(\frac{\partial^{2} w}{\partial x^{2}}+s \frac{\partial^{2} w}{\partial y^{2}}\right) \\
& -\frac{a^{2}}{6} P(s+1) \frac{\partial^{4} w}{\partial x^{2} \partial y^{2}}-\frac{a^{2}}{12} P\left(\frac{\partial^{4} w}{\partial x^{4}}+s \frac{\partial^{4} w}{\partial y^{4}}\right)=0
\end{aligned}
$$

This equation containing fourth or less derivative terms following a same contribution, namely $x$ and $y$, it is called fourth-order continualized model. Its beam counterpart is the fourth-order continualized model, as developed in Sect. 2.2.

Thus, considering two different continualization approaches, it leads to two different but similar sixth-order governing differential equation. Again, Eq. (133) requires six boundary conditions which may be obtained from the variational principle, through the following postulated energies $U, T$ and $W$ :

$$
\begin{aligned}
U(w)= & \frac{1}{2} \iint_{\mathfrak{D}} D\left[\left(\frac{\partial^{2} w}{\partial x^{2}}\right)^{2}+2 \nu \frac{\partial^{2} w}{\partial x^{2}} \frac{\partial^{2} w}{\partial y^{2}}+\left(\frac{\partial^{2} w}{\partial y^{2}}\right)^{2}+2(1-\nu)\left(\frac{\partial^{2} w}{\partial x \partial y}\right)^{2}\right. \\
& \left.+\frac{a^{2}}{3}\left(\left(\frac{\partial^{3} w}{\partial x^{2} \partial y}\right)^{2}+\left(\frac{\partial^{3} w}{\partial x \partial y^{2}}\right)^{2}\right)\right] \mathrm{d} x \mathrm{~d} y \\
T(w)= & \frac{1}{2} \iint_{\mathfrak{D}} m_{0} \omega^{2}\left[w^{2}+\frac{a^{2}}{6}\left(\left(\frac{\partial w}{\partial x}\right)^{2}+\left(\frac{\partial w}{\partial y}\right)^{2}\right)\right] \mathrm{d} x \mathrm{~d} y \\
W(w)= & \frac{1}{2} \iint_{\mathfrak{D}} P\left[\left(\frac{\partial w}{\partial x}\right)^{2}+s\left(\frac{\partial w}{\partial y}\right)^{2}+\frac{a^{2}}{12}\left(\left(\frac{\partial^{2} w}{\partial x^{2}}\right)^{2}+s\left(\frac{\partial^{2} w}{\partial y^{2}}\right)^{2}\right)\right. \\
& \left.+\frac{a^{2}}{6}(s+1) \frac{\partial^{2} w}{\partial x^{2}} \frac{\partial^{2} w}{\partial y^{2}}\right] \mathrm{d} x \mathrm{~d} y
\end{aligned}
$$

Thus, applying the Hamilton principle $\delta(U-T-W)$, we obtain Eq. (133) with the natural boundary conditions written under the line integral form:

$$
\oint_{\Gamma} I(x, y)=0
$$

where $\Gamma$ is the boundary of the plate and $I$ is defined as follows

$$
\begin{aligned}
I(x, y)= & \frac{a^{2}}{3} D \frac{\partial^{3} w}{\partial x^{2} \partial y} \delta\left(\frac{\partial^{2} w}{\partial x^{2}}\right) \mathrm{d} x+\frac{a^{2}}{3} D \frac{\partial^{3} w}{\partial y^{2} \partial x} \delta\left(\frac{\partial^{2} w}{\partial y^{2}}\right) \mathrm{d} y \\
& +\left\{D\left(v \frac{\partial^{2} w}{\partial x^{2}}+\frac{\partial^{2} w}{\partial y^{2}}\right)-D \frac{a^{2}}{3} \frac{\partial^{4} w}{\partial x^{2} \partial y^{2}}-\frac{a^{2}}{12} P\left[s \frac{\partial^{2} w}{\partial y^{2}}+(1+s) \frac{\partial^{2} w}{\partial x^{2}}\right]\right\} \delta\left(\frac{\partial w}{\partial y}\right) \mathrm{d} x \\
& +\left\{D\left(\frac{\partial^{2} w}{\partial x^{2}}+v \frac{\partial^{2} w}{\partial y^{2}}\right)-D \frac{a^{2}}{3} \frac{\partial^{4} w}{\partial x^{2} \partial y^{2}}-\frac{a^{2}}{12} P\left[\frac{\partial^{2} w}{\partial x^{2}}+(1+s) \frac{\partial^{2} w}{\partial y^{2}}\right]\right\} \delta\left(\frac{\partial w}{\partial x}\right) \mathrm{d} y \\
& -\left\{D\left[\frac{\partial^{3} w}{\partial x^{3}}+\frac{\partial^{3} w}{\partial x \partial y^{2}}-\frac{a^{2}}{3} \frac{\partial^{5} w}{\partial x^{3} \partial y^{2}}\right]\right.
\end{aligned}
$$




$$
\begin{aligned}
& \left.+P \frac{\partial w}{\partial x}-\frac{a^{2}}{12} P\left[\frac{\partial^{3} w}{\partial x^{3}}+(1+s) \frac{\partial^{3} w}{\partial x \partial y^{2}}\right]+\frac{a^{2}}{6} m_{0} \omega^{2} \frac{\partial w}{\partial y}\right\} \delta w \mathrm{~d} y \\
& -\left\{D\left[\frac{\partial^{3} w}{\partial y^{3}}+\frac{\partial^{3} w}{\partial x^{2} \partial y}-\frac{a^{2}}{3} \frac{\partial^{5} w}{\partial x^{2} \partial y^{3}}\right]\right. \\
& \left.+s P \frac{\partial w}{\partial y}-\frac{a^{2}}{12} P\left[s \frac{\partial^{3} w}{\partial y^{3}}+(1+s) \frac{\partial^{3} w}{\partial x^{2} \partial y}\right]+\frac{a^{2}}{6} m_{0} \omega^{2} \frac{\partial w}{\partial x}\right\} \delta w \mathrm{~d} x
\end{aligned}
$$

The solution is provided for simply supported plate. In view of boundary conditions, similarly to Eq. (111) the solution may be expressed as

$$
w=w_{0} \sin \frac{m \pi x}{L} \sin \frac{n \pi y}{\alpha L}
$$

Setting $\Omega^{2}=\omega^{2} m(\alpha L)^{4} / D$ and $\lambda=\omega^{2} m(\alpha L)^{2} / D$ and substituting Eq. (139) into Eq. (191), the solution is given by:

$$
\begin{aligned}
\Omega^{2}= & \frac{\left(\frac{\alpha L}{a}\right)^{2}\left(n^{2}+m^{2} \alpha^{2}\right)^{2} \pi^{4}-e_{0}^{2} \pi^{6}\left[(\alpha m)^{6}-(\alpha m)^{4} n^{2}-(\alpha m)^{2} n^{4}+n^{6}\right]}{\left[\left(\frac{\alpha L}{a}\right)^{2}+e_{0}^{2} \pi^{2}\left[(\alpha m)^{2}+n^{2}\right]\right]} \\
& -\lambda \frac{\pi^{2}\left[e_{0}^{2} \pi^{2}(\alpha m n)^{2}(1+s)+\left(\frac{\alpha L}{a}\right)^{2}\left[(\alpha m)^{2}+s n^{2}\right]\right]}{\left[\left(\frac{\alpha L}{a}\right)^{2}+\left(1+\gamma_{3}\right) e_{0}^{2} \pi^{2}\left[(\alpha m)^{2}+n^{2}\right]\right]}
\end{aligned}
$$

with $e_{0}^{2}=1 / 12$ for the continualization processes. In the static case, $\Omega^{2}=0$, assuming a half sine wave in the y direction $(n=1)$, the buckling load is

$$
\lambda=\min _{(m, n)} \pi^{2}\left\{\frac{\left(\frac{\alpha L}{a}\right)^{2}\left(1+m^{2} \alpha^{2}\right)^{2}-e_{0}^{2} \pi^{2}\left[(\alpha m)^{6}-(\alpha m)^{4}-(\alpha m)^{2}+1\right]}{\left[e_{0}^{2} \pi^{2}(\alpha m)^{2}(1+s)+\left(\frac{\alpha L}{a}\right)^{2}\left[(\alpha m)^{2}+s\right]\right]}\right\}
$$

Next, we shall consider the particular case of a uniaxial load, i.e. $s=0$. The squared natural frequency becomes:

$$
\Omega^{2}=\frac{\left(\frac{\alpha L}{a}\right)^{2}\left(n^{2}+m^{2} \alpha^{2}\right)^{2} \pi^{4}-e_{0}^{2} \pi^{6}\left[(\alpha m)^{6}-(\alpha m)^{4} n^{2}-(\alpha m)^{2} n^{4}+n^{6}\right]-\lambda \pi^{2}(\alpha m)^{2}\left[e_{0}^{2} \pi^{2} n^{2}+\left(\frac{\alpha L}{a}\right)^{2}\right]}{\left[\left(\frac{\alpha L}{a}\right)^{2}+e_{0}^{2} \pi^{2}\left[(\alpha m)^{2}+n^{2}\right]\right]}
$$

Furthermore, in free vibration, the frequency is calculated from $\lambda=0$ in Eq. (142)

$$
\Omega^{2}=\frac{\left(\frac{\alpha L}{a}\right)^{2}\left(n^{2}+m^{2} \alpha^{2}\right)^{2} \pi^{4}}{\left[\left(\frac{\alpha L}{a}\right)^{2}+e_{0}^{2} \pi^{2}\left[(\alpha m)^{2}+n^{2}\right]\right]}-e_{0}^{2} \pi^{6} \frac{\left[(\alpha m)^{6}-(\alpha m)^{4} n^{2}-(\alpha m)^{2} n^{4}+n^{6}\right]}{\left[\left(\frac{\alpha L}{a}\right)^{2}+e_{0}^{2} \pi^{2}\left[(\alpha m)^{2}+n^{2}\right]\right]}
$$

As explained above; the small length scale coefficient is, by definition, constant $\left(e_{0}=1 / \sqrt{12}\right)$ and structural independent.

\subsection{Sixth-order continualized model}

An alternative continualized model can be derived, by using another continualization scheme. Multiplying Eqs. (120) and (121) by $\left[1-\left(a^{2} / 12\right) \partial^{2} / \partial x^{2}\right]\left[1-\left(a^{2} / 12\right) \partial^{2} / \partial y^{2}\right]$ and Eq. (122) by $\left[1-\left(a^{2} / 24\right) \partial^{2} / \partial x^{2}\right][1-$ $\left.\left(a^{2} / 24\right) \partial^{2} / \partial y^{2}\right]$, one obtains

$$
M_{x}-\frac{a^{2}}{12}\left(\frac{\partial^{2}}{\partial x^{2}}+\frac{\partial^{2}}{\partial y^{2}}\right) M_{x}=D\left[\frac{\partial^{2} w}{\partial x^{2}}+v \frac{\partial^{2} w}{\partial y^{2}}-\frac{a^{2}}{12}(1+v) \frac{\partial^{4} w}{\partial x^{2} \partial y^{2}}\right]+o\left(a^{2}\right)
$$




$$
\begin{aligned}
M_{y}-\frac{a^{2}}{12}\left(\frac{\partial^{2}}{\partial x^{2}}+\frac{\partial^{2}}{\partial y^{2}}\right) M_{y} & =D\left[\frac{\partial^{2} w}{\partial y^{2}}+v \frac{\partial^{2} w}{\partial x^{2}}-\frac{a^{2}}{12}(1+v) \frac{\partial^{4} w}{\partial x^{2} \partial y^{2}}\right]+o\left(a^{2}\right) \\
M_{x y}-\frac{a^{2}}{24}\left(\frac{\partial^{2}}{\partial x^{2}}+\frac{\partial^{2}}{\partial y^{2}}\right) M_{x y} & =D(1-v) \frac{\partial^{2}}{\partial x \partial y} w+o\left(a^{2}\right)
\end{aligned}
$$

Eqs. (144)-(146) are close to Eringen's nonlocal elasticity Eqs. (74)-(76), except the last nonlocal underlined term. Using Eqs. (144)-(146) into Eq. (123), multiplying by $\left[1-\left(e_{0} a\right)^{2} \partial^{2} / \partial x^{2}\right]\left[1-\left(e_{0} a\right)^{2} \partial^{2} / \partial y^{2}\right]$ one obtains the approximation

$$
\begin{aligned}
& D\left[\frac{\partial^{4} w}{\partial x^{4}}+2 \frac{\partial^{4} w}{\partial x^{2} \partial y^{2}}+\frac{\partial^{4} w}{\partial y^{4}}+\frac{a^{2}}{12}\left(\frac{\partial^{6} w}{\partial x^{6}}-\frac{\partial^{6} w}{\partial x^{4} \partial y^{2}}-\frac{\partial^{6} w}{\partial x^{2} \partial y^{4}}+\frac{\partial^{6} w}{\partial y^{6}}\right)\right] \\
& \quad-\left[1-\frac{a^{2}}{12}\left(\frac{\partial^{2}}{\partial x^{2}}+\frac{\partial^{2}}{\partial y^{2}}\right)\right] m_{0} \omega^{2} w+P\left(\frac{\partial^{2} w}{\partial x^{2}}+s \frac{\partial^{2} w}{\partial y^{2}}\right)-\frac{a^{2}}{12}(1+s) P \frac{\partial^{4} w}{\partial x^{2} \partial y^{2}}=0
\end{aligned}
$$

Equation (147) can also be derived from the continualization of the discrete governing difference equation Eq. (105). We start from Eq. (123), equation based on Padé approximants and we use the Taylor expansion, at the second order in $a$ :

$$
\begin{aligned}
& D\left[\left(1+\frac{a^{2}}{6} \frac{\partial^{2}}{\partial x^{2}}\right) \frac{\partial^{4} w}{\partial x^{4}}+2\left(1+\frac{a^{2}}{12} \frac{\partial^{2}}{\partial x^{2}}\right)\left(1+\frac{a^{2}}{12} \frac{\partial^{2}}{\partial y^{2}}\right) \frac{\partial^{4} w}{\partial x^{2} \partial y^{2}}+\left(1+\frac{a^{2}}{6} \frac{\partial^{2}}{\partial y^{2}}-\frac{a^{4}}{144} \frac{\partial^{4}}{\partial y^{4}}\right) \frac{\partial^{4} w}{\partial y^{4}}\right] \\
& -m_{0} \omega^{2} w+P\left[\left(1+\frac{a^{2}}{12} \frac{\partial^{2}}{\partial x^{2}}\right) \frac{\partial^{2} w}{\partial x^{2}}+s\left(1+\frac{a^{2}}{12} \frac{\partial^{2}}{\partial y^{2}}\right) \frac{\partial^{2} w}{\partial y^{2}}\right]+o\left(a^{4}\right)=0
\end{aligned}
$$

Multiplying by $\left[1-\left(e_{0} a\right)^{2}\left(\partial^{2} / \partial x^{2}+\partial^{2} / \partial y^{2}\right)\right]$

$$
\begin{aligned}
& D\left[\frac{\partial^{4} w}{\partial x^{4}}+2 \frac{\partial^{4} w}{\partial x^{2} \partial y^{2}}+\frac{\partial^{4} w}{\partial y^{4}}+\frac{a^{2}}{12}\left(\frac{\partial^{6} w}{\partial x^{6}}-\frac{\partial^{6} w}{\partial x^{4} \partial y^{2}}-\frac{\partial^{6} w}{\partial x^{2} \partial y^{4}}+\frac{\partial^{6} w}{\partial y^{6}}\right)\right. \\
& \left.-2\left(e_{0} a\right)^{4}\left(2 \frac{\partial^{8} w}{\partial x^{6} \partial y^{2}}+3 \frac{\partial^{8} w}{\partial x^{4} \partial y^{4}}+2 \frac{\partial^{8} w}{\partial x^{2} \partial y^{6}}\right)\right] \\
& -\left[1-\frac{a^{2}}{12}\left(\frac{\partial^{2}}{\partial x^{2}}+\frac{\partial^{2}}{\partial y^{2}}\right)\right] m_{0} \omega^{2} w+P\left(\frac{\partial^{2} w}{\partial x^{2}}+s \frac{\partial^{2} w}{\partial y^{2}}\right)-\frac{a^{2}}{12}(1+s) P \frac{\partial^{4} w}{\partial x^{2} \partial y^{2}} \\
& +\frac{a^{4}}{144} P\left(\frac{\partial^{2}}{\partial x^{2}}+\frac{\partial^{2}}{\partial y^{2}}\right)\left(\frac{\partial^{4} w}{\partial x^{4}}+s \frac{\partial^{4} w}{\partial y^{4}}\right)+o\left(a^{4}\right)=0
\end{aligned}
$$

Neglecting the higher-order terms in a, we obtain Eq. (147). It is also obtainable via variational formulation, using the following bending strain energy, kinetic energy and work done by axial forces:

$$
\begin{aligned}
U(w)= & \frac{1}{2} \iint_{\mathfrak{D}} D\left[\left(\frac{\partial^{2} w}{\partial x^{2}}\right)^{2}+2 \nu \frac{\partial^{2} w}{\partial x^{2}} \frac{\partial^{2} w}{\partial y^{2}}+\left(\frac{\partial^{2} w}{\partial y^{2}}\right)^{2}\right. \\
& +2(1-\nu)\left(\frac{\partial^{2} w}{\partial x \partial y}\right)^{2}-\frac{a^{2}}{12}\left(\left(\frac{\partial^{3} w}{\partial x^{3}}\right)^{2}+\left(\frac{\partial^{3} w}{\partial y^{3}}\right)^{2}\right) \\
& \left.+\frac{a^{2}}{12}\left(\left(\frac{\partial^{3} w}{\partial x^{2} \partial y}\right)^{2}+\left(\frac{\partial^{3} w}{\partial x \partial y^{2}}\right)^{2}\right)\right] \mathrm{d} x \mathrm{~d} y \\
T(w)= & \frac{1}{2} \iint_{\mathfrak{D}} m_{0} \omega^{2}\left[w^{2}+\frac{a^{2}}{12}\left(\left(\frac{\partial w}{\partial x}\right)^{2}+\left(\frac{\partial w}{\partial y}\right)^{2}\right)\right] \mathrm{d} x \mathrm{~d} y \\
W(w)= & \frac{1}{2} \iint_{\mathfrak{D}} P\left[\left(\frac{\partial w}{\partial x}\right)^{2}+s\left(\frac{\partial w}{\partial y}\right)^{2}+\frac{a^{2}}{12}(1+s) \frac{\partial^{2} w}{\partial x^{2}} \frac{\partial^{2} w}{\partial y^{2}}\right] \mathrm{d} x \mathrm{~d} y
\end{aligned}
$$


where $\mathfrak{D}$ is the area of the mid-surface of the plate. In this model, the nonlocality does not affect the work. Thus, applying the Hamilton principle, it yields Eq. (133) with the natural boundary conditions given by (137) and $I$ defined as follows

$$
\begin{aligned}
I(x, y, t)= & \frac{a^{2}}{12} D\left[\frac{\partial^{3} w}{\partial x^{2} \partial y} \frac{\partial^{2} \delta w}{\partial x^{2}}-\frac{\partial^{3} w}{\partial y^{3}} \frac{\partial^{2} \delta w}{\partial y^{2}}\right] \mathrm{d} x+\frac{a^{2}}{12} D\left[\frac{\partial^{3} w}{\partial x \partial y^{2}} \frac{\partial^{2} \delta w}{\partial y^{2}}-\frac{\partial^{3} w}{\partial x^{3}} \frac{\partial^{2} \delta w}{\partial x^{2}}\right] \mathrm{d} y \\
& +\left\{D\left(v \frac{\partial^{2} w}{\partial x^{2}}+\frac{\partial^{2} w}{\partial y^{2}}\right)-D \frac{a^{2}}{12}\left(\frac{\partial^{4} w}{\partial x^{2} \partial y^{2}}-\frac{\partial^{4} w}{\partial y^{4}}\right)-\frac{a^{2}}{24} P(1+s) \frac{\partial^{2} w}{\partial x^{2}}\right\} \delta\left(\frac{\partial w}{\partial y}\right) \mathrm{d} x \\
& +D(1-v) \frac{\partial^{2} w}{\partial x \partial y} \delta\left(\frac{\partial w}{\partial x}\right) \mathrm{d} x+D(1-v) \frac{\partial^{2} w}{\partial x \partial y} \delta\left(\frac{\partial w}{\partial y}\right) \mathrm{d} y \\
& +\left\{D\left(\frac{\partial^{2} w}{\partial x^{2}}+v \frac{\partial^{2} w}{\partial y^{2}}\right)-D \frac{a^{2}}{12}\left(\frac{\partial^{4} w}{\partial x^{2} \partial y^{2}}-\frac{\partial^{4} w}{\partial x^{4}}\right)-\frac{a^{2}}{24} P(1+s) \frac{\partial^{2} w}{\partial y^{2}}\right\} \delta\left(\frac{\partial w}{\partial x}\right) \mathrm{d} y \\
& -\left\{D\left[\frac{\partial^{3} w}{\partial x^{3}}+\frac{\partial^{3} w}{\partial x \partial y^{2}}+\frac{a^{2}}{12}\left(\frac{\partial^{5} w}{\partial x^{5}}-\frac{\partial^{5} w}{\partial x^{3} \partial y^{2}}\right)\right]\right. \\
& \left.+P\left(\frac{\partial w}{\partial x}-\frac{a^{2}}{24}(1+s) \frac{\partial^{3} w}{\partial x \partial y^{2}}\right)+\frac{a^{2}}{12} m_{0} \omega^{2} \frac{\partial w}{\partial x}\right\} \delta w \mathrm{~d} y \\
& -\left\{D\left[\frac{\partial^{3} w}{\partial y^{3}}+\frac{\partial^{3} w}{\partial x^{2} \partial y}+\frac{a^{2}}{12}\left(\frac{\partial^{5} w}{\partial y^{5}}-\frac{\partial^{5} w}{\partial x^{2} \partial y^{3}}\right)\right]\right. \\
& \left.+P\left(s \frac{\partial w}{\partial y}-\frac{a^{2}}{24}(1+s) \frac{\partial^{3} w}{\partial x^{2} \partial y}\right)+\frac{a^{2}}{12} m_{0} \omega^{2} \frac{\partial w}{\partial y}\right\} \delta w \mathrm{~d} x
\end{aligned}
$$

In contrast to local models, two additional boundary conditions appear.

In view of the simply supported boundary conditions, the substitution of Eq. (139) into Eq. (147) yields the following solution

$$
\begin{aligned}
\Omega^{2}= & \frac{\left(\frac{\alpha L}{a}\right)^{2}\left(n^{2}+m^{2} \alpha^{2}\right)^{2} \pi^{4}+4 e_{0}^{2} \pi^{6}(\alpha m)^{2} n^{2}\left[(\alpha m)^{2}+n^{2}\right]}{\left[\left(\frac{\alpha L}{a}\right)^{2}+2 e_{0}^{2} \pi^{2}\left[(\alpha m)^{2}+n^{2}\right]\right]} \\
& -\frac{\lambda \pi^{2}\left[2 e_{0}^{2} \pi^{2}(\alpha m n)^{2}(1+s)+e_{0}^{2} \pi^{2}\left[(\alpha m)^{4}+s n^{4}\right]+\left(\frac{\alpha L}{a}\right)^{2}\left[(\alpha m)^{2}+s n^{2}\right]\right]}{\left[\left(\frac{\alpha L}{a}\right)^{2}+2 e_{0}^{2} \pi^{2}\left[(\alpha m)^{2}+n^{2}\right]\right]}
\end{aligned}
$$

with $e_{0}^{2}=1 / 12$ for the continualization processes. In the static case, $\Omega^{2}=0$, assuming a half sine wave in the $\mathrm{y}$ direction $(n=1)$, the buckling load parameter is

$$
\lambda=\min _{(m, n)} \pi^{4}\left\{\frac{\left(\frac{\alpha L}{a}\right)^{2}\left(1+m^{2} \alpha^{2}\right)^{2}+4 e_{0}^{2}(\alpha m)^{2}\left(1+m^{2} \alpha^{2}\right)}{\left[2 e_{0}^{2} \pi^{2}(\alpha m)^{2}(1+s)+e_{0}^{2} \pi^{2}\left[(\alpha m)^{4}+s\right]+\left(\frac{\alpha L}{a}\right)^{2}\left[(\alpha m)^{2}+s\right]\right]}\right\}
$$

For the particular case of a uniaxial load, i.e. $s=0$, the squared natural frequency parameter becomes:

$$
\begin{aligned}
\Omega^{2}= & \frac{\left(\frac{\alpha L}{a}\right)^{2}\left(n^{2}+m^{2} \alpha^{2}\right)^{2} \pi^{4}}{\left[\left(\frac{\alpha L}{a}\right)^{2}+2 e_{0}^{2} \pi^{2}\left[(\alpha m)^{2}+n^{2}\right]\right]}-\lambda \pi^{2}(\alpha m)^{2} \frac{2 e_{0}^{2} \pi^{2} n^{2}+e_{0}^{2} \pi^{2}\left[(\alpha m)^{2}\right]+\left(\frac{\alpha L}{a}\right)^{2}}{\left[\left(\frac{\alpha L}{a}\right)^{2}+2 e_{0}^{2} \pi^{2}\left[(\alpha m)^{2}+n^{2}\right]\right]} \\
& +\frac{4 e_{0}^{2} \pi^{6}(\alpha m)^{2} n^{2}\left[(\alpha m)^{2}+n^{2}\right]}{\left[\left(\frac{\alpha L}{a}\right)^{2}+2 e_{0}^{2} \pi^{2}\left[(\alpha m)^{2}+n^{2}\right]\right]}
\end{aligned}
$$

Furthermore, in free vibration, the frequency is calculated from $\lambda=0$

$$
\Omega^{2}=\frac{\left(\frac{\alpha L}{a}\right)^{2}\left(n^{2}+m^{2} \alpha^{2}\right)^{2} \pi^{4}}{\left[\left(\frac{\alpha L}{a}\right)^{2}+2 e_{0}^{2} \pi^{2}\left[(\alpha m)^{2}+n^{2}\right]\right]}+4 e_{0}^{2} \pi^{6}(\alpha m)^{2} n^{2} \frac{\left[(\alpha m)^{2}+n^{2}\right]}{\left[\left(\frac{\alpha L}{a}\right)^{2}+2 e_{0}^{2} \pi^{2}\left[(\alpha m)^{2}+n^{2}\right]\right]}
$$


As for the fourth-order continualized model, the small length scale coefficient is constant and structural problem independent.

\subsection{Fourth-order phenomenological model: nonlocal Eringen's theory}

According to Eringen's nonlocal theory [14] applied for plates, the nonlocal versions of moment-curvature relations Eqs. (101)-(103) are postulated as follows [20,49]:

$$
\begin{aligned}
& M_{x x}-\left(e_{0} a\right)^{2}\left(\frac{\partial^{2} M_{x x}}{\partial x^{2}}+\frac{\partial^{2} M_{x x}}{\partial y^{2}}\right)=D\left(\frac{\partial^{2} w}{\partial x^{2}}+\nu \frac{\partial^{2} w}{\partial y^{2}}\right) \\
& M_{y y}-\left(e_{0} a\right)^{2}\left(\frac{\partial^{2} M_{y y}}{\partial x^{2}}+\frac{\partial^{2} M_{y y}}{\partial y^{2}}\right)=D\left(\frac{\partial^{2} w}{\partial y^{2}}+\nu \frac{\partial^{2} w}{\partial x^{2}}\right) \\
& M_{x y}-\left(e_{0} a\right)^{2}\left(\frac{\partial^{2} M_{x y}}{\partial x^{2}}+\frac{\partial^{2} M_{x y}}{\partial y^{2}}\right)=D(1-\nu) \frac{\partial^{2} w}{\partial x \partial y}
\end{aligned}
$$

By substituting Eqs. (158)-(160) into the local Eq. (104), the governing differential equation is obtained

$$
\begin{aligned}
& D\left(\frac{\partial^{4} w}{\partial x^{4}}+2 \frac{\partial^{4} w}{\partial x^{2} \partial y^{2}}+\frac{\partial^{4} w}{\partial y^{4}}\right)-m_{0} \omega^{2}\left[w-\left(e_{0} a\right)^{2}\left(\frac{\partial^{2} w}{\partial x^{2}}+\frac{\partial^{2} w}{\partial y^{2}}\right)\right] \\
& -\left(e_{0} a\right)^{2} \frac{\partial^{4} w}{\partial x^{2} \partial y^{2}} P(1+s)-\left(e_{0} a\right)^{2} P\left(\frac{\partial^{4} w}{\partial x^{4}}+s \frac{\partial^{4} w}{\partial y^{4}}\right)+P\left(\frac{\partial^{2} w}{\partial x^{2}}+s \frac{\partial^{2} w}{\partial y^{2}}\right)=0
\end{aligned}
$$

Eq. (161) may be obtained variationally using the following bending strain energy, kinetic energy and work done by axial forces:

$$
\begin{aligned}
U(w)= & \frac{1}{2} \iint_{\mathfrak{D}} D\left[\left(\frac{\partial^{2} w}{\partial x^{2}}\right)^{2}+2 \nu \frac{\partial^{2} w}{\partial x^{2}} \frac{\partial^{2} w}{\partial y^{2}}+\left(\frac{\partial^{2} w}{\partial y^{2}}\right)^{2}+2(1-\nu)\left(\frac{\partial^{2} w}{\partial x \partial y}\right)^{2}\right] \mathrm{d} x \mathrm{~d} y \\
T(w)= & \frac{1}{2} \iint_{\mathfrak{D}} m_{0} \omega^{2}\left[w^{2}+\left(e_{0} a\right)^{2}\left(\left(\frac{\partial w}{\partial x}\right)^{2}+\left(\frac{\partial w}{\partial y}\right)^{2}\right)\right] \mathrm{d} x \mathrm{~d} y \\
W(w)= & \frac{1}{2} \iint_{\mathfrak{D}} P\left[\left(\frac{\partial w}{\partial x}\right)^{2}+s\left(\frac{\partial w}{\partial y}\right)^{2}+\left(e_{0} a\right)^{2}\left(\frac{\partial^{2} w}{\partial x^{2}}\right)^{2}+\left(e_{0} a\right)^{2} s\left(\frac{\partial^{2} w}{\partial y^{2}}\right)^{2}\right. \\
& \left.+\left(e_{0} a\right)^{2}(s+1) \frac{\partial^{2} w}{\partial x^{2}} \frac{\partial^{2} w}{\partial y^{2}}\right] \mathrm{d} x \mathrm{~d} y
\end{aligned}
$$

Equations (162)-(164) match with the results of Chakraverty and Behera [50] and the functional given by Phadikar and Pradhan [51]. It is worth noting that the nonlocal effect does not affect the bending strain energy.

The kinetic energy is different from the one given by Adali $[52,53]$ :

$$
T(w)=\frac{1}{2} \iint_{\mathfrak{D}} m_{0} \omega^{2}\left[w^{2}-\left(e_{0} a\right)^{2} w\left(\frac{\partial^{2} w}{\partial x^{2}}+\frac{\partial^{2} w}{\partial x^{2}}\right)\right] \mathrm{d} x \mathrm{~d} y
$$

Although they lead to the same governing differential equation, they do not provide the same boundary conditions. We retain the kinetic energy given by Chakraverty and Behera [50], definite positive.

Thus, by applying the Hamilton principle, we get Eq. (161) with the natural boundary conditions given by (137) and $I$ defined as follows:

$$
\begin{aligned}
I(x, y)= & \left\{D\left(v \frac{\partial^{2} w}{\partial x^{2}}+\frac{\partial^{2} w}{\partial y^{2}}\right)-\frac{\left(e_{0} a\right)^{2} P}{2}\left[2 s \frac{\partial^{2} w}{\partial y^{2}}+(1+s) \frac{\partial^{2} w}{\partial x^{2}}\right]\right\} \delta\left(\frac{\partial w}{\partial y}\right) \mathrm{d} x \\
& +\left\{D\left(\frac{\partial^{2} w}{\partial x^{2}}+v \frac{\partial^{2} w}{\partial y^{2}}\right)-\frac{\left(e_{0} a\right)^{2} P}{2}\left[2 \frac{\partial^{2} w}{\partial x^{2}}+(1+s) \frac{\partial^{2} w}{\partial y^{2}}\right]\right\} \delta\left(\frac{\partial w}{\partial x}\right) \mathrm{d} y
\end{aligned}
$$




$$
\begin{aligned}
& -\left\{D\left[\frac{\partial^{3} w}{\partial x^{3}}+\frac{\partial^{3} w}{\partial x \partial y^{2}}\right]+P \frac{\partial w}{\partial x}-\frac{\left(e_{0} a\right)^{2} P}{2}\left[2 \frac{\partial^{3} w}{\partial x^{3}}+(1+s) \frac{\partial^{3} w}{\partial x \partial y^{2}}\right]\right. \\
& \left.+\left(e_{0} a\right)^{2} m_{0} \omega^{2} \frac{\partial w}{\partial x}\right\} \delta w \mathrm{~d} y \\
& -\left\{D\left[\frac{\partial^{3} w}{\partial y^{3}}+\frac{\partial^{3} w}{\partial x^{2} \partial y}\right]+s P \frac{\partial w}{\partial y}-\frac{\left(e_{0} a\right)^{2} P}{2}\left[2 s \frac{\partial^{3} w}{\partial y^{3}}+(1+s) \frac{\partial^{3} w}{\partial x^{2} \partial y}\right]\right. \\
& \left.+\left(e_{0} a\right)^{2} m_{0} \omega^{2} \frac{\partial w}{\partial y}\right\} \delta w \mathrm{~d} x
\end{aligned}
$$

As for the beam case, the boundary conditions obtained through the variational principle and the one issued of the principle of virtual work may differ.

In dynamics, the dynamic contribution in the boundary conditions differ between the traditional nonlocal Eringen's model and the conditions obtained through the phenomenological approach Eqs. (60) and (61). In view of the simply supported boundary conditions, the substitution of Eq. (139) into Eq. (166) furnishes the following solution

$$
\Omega^{2}=\frac{\left(\frac{\alpha L}{a}\right)^{2}\left(n^{2}+m^{2} \alpha^{2}\right)^{2} \pi^{4}-\lambda \pi^{2}\left[e_{0}^{2} \pi^{2}(\alpha m n)^{2}(1+s)+e_{0}^{2} \pi^{2}\left[(\alpha m)^{4}+s n^{4}\right]+\left(\frac{\alpha L}{a}\right)^{2}\left[(\alpha m)^{2}+s n^{2}\right]\right]}{\left[\left(\frac{\alpha L}{a}\right)^{2}+e_{0}^{2} \pi^{2}\left[(\alpha m)^{2}+n^{2}\right]\right]}
$$

For $s=1$, the solution coincide with the one derived by Zhang et al. [48].

In statics, the buckling load parameter is

$$
\lambda=\min _{(m, n)} \pi^{4}\left\{\frac{\left(\frac{\alpha L}{a}\right)^{2}\left(1+m^{2} \alpha^{2}\right)^{2}}{\left[e_{0}^{2} \pi^{2}(\alpha m)^{2}(1+s)+e_{0}^{2} \pi^{2}\left[(\alpha m)^{4}+s\right]+\left(\frac{\alpha L}{a}\right)^{2}\left[(\alpha m)^{2}+s\right]\right]}\right\}
$$

This result coincides with the one obtained by Zhang et al. [48]. For a uniaxial load, i.e. $s=0$, the squared natural frequency parameter is:

$$
\Omega^{2}=\frac{\left(\frac{\alpha L}{a}\right)^{2}\left(n^{2}+m^{2} \alpha^{2}\right)^{2} \pi^{4}}{\left(\frac{\alpha L}{a}\right)^{2}+e_{0}^{2} \pi^{2}\left(n^{2}+m^{2} \alpha^{2}\right)}-\lambda \pi^{2}(\alpha m)^{2} \frac{e_{0}^{2} \pi^{2} n^{2}+e_{0}^{2} \pi^{2}(\alpha m)^{2}+\left(\frac{\alpha L}{a}\right)^{2}}{\left(\frac{\alpha L}{a}\right)^{2}+e_{0}^{2} \pi^{2}\left(n^{2}+m^{2} \alpha^{2}\right)}
$$

Furthermore, in free vibration, for $\lambda=0$, the frequency is:

$$
\Omega^{2}=\frac{\left(\frac{\alpha L}{a}\right)^{2}\left(n^{2}+m^{2} \alpha^{2}\right)^{2} \pi^{4}}{\left(\frac{\alpha L}{a}\right)^{2}+e_{0}^{2} \pi^{2}\left(n^{2}+m^{2} \alpha^{2}\right)}
$$

Considering a phenomenological approach, the small length scale coefficient turns out to be not a constant. It depends on the initial load $(P$ or $\lambda)$, the vibration frequency $\Omega$, the vibration/buckling mode $(m, n)$, the material (the internal characteristic $a$ ) and the geometry (aspect ratio $\alpha$ and one of the length of the beam L) [20]. Thus, it has to be calibrated. In a similar manner to the investigation of nonlocal beam models, by relating the microstructured beam-grid model to the nonlocal rectangular plate, the length of rigid element can be interpreted as the internal characteristic length scale. As in beam problems, the small length scale coefficient is obtained by comparing the solutions of a microstructured beam-grid model and a nonlocal rectangular plate model. More especially, by equating the buckling stress from Eqs. (116) and (168), with the assumption of having one-half wave in the $y$-direction $(n=1)$, the small length scale coefficient may be expressed as

$$
\frac{1}{e_{0}^{2}}=\frac{\frac{2 n_{y}^{2}\left(\cos \frac{\alpha m \pi}{n_{y}}+\cos \frac{\pi}{n_{y}}-2\right)^{2}}{1+s-\cos \frac{\alpha m \pi}{n_{y}}-s \cos \frac{\pi}{n_{y}}} \pi^{2}\left[(\alpha m)^{2}(1+s)+\left[(\alpha m)^{4}+s\right]\right]}{n_{y}^{2} \pi^{2}\left(1+m^{2} \alpha^{2}\right)^{2}-\frac{2 n_{y}^{4}\left(\cos \frac{\alpha m \pi}{n_{y}}+\cos \frac{\pi}{n_{y}}-2\right)^{2}}{1+s-\cos \frac{\alpha m \pi}{n_{y}}-s \cos \frac{\pi}{n_{y}}}\left[(\alpha m)^{2}+s\right]}
$$



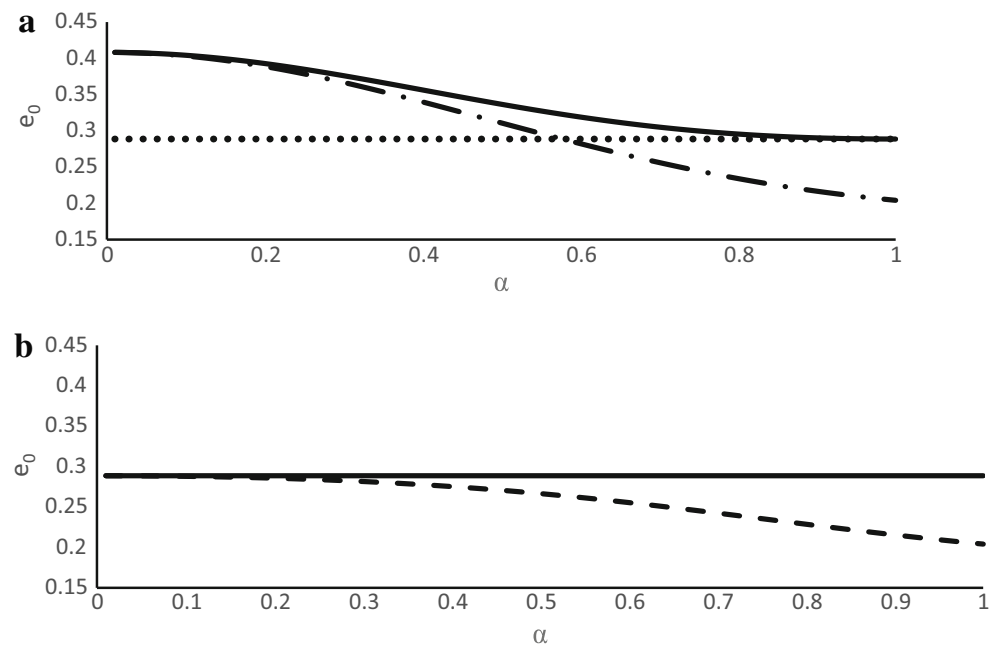

Fig. 5 Variation of calibrated small length scale coefficient calculated in static case (dashed line) and free vibration case (continuous line) with respect to aspect ratio, considering a the fourth-order phenomenological model (Eringen's model) and the continualized model (dotted line) and $\mathbf{b}$ the sixth-order phenomenological model

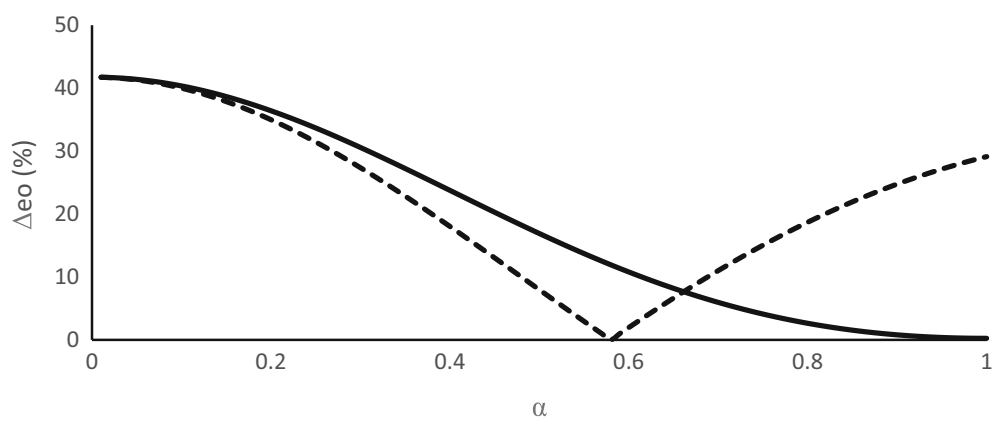

Fig. 6 Gap between the small length scale coefficient calibrated in the fourth-order phenomenological model (Eringen's model) and the exact one obtained in the continualized model in the static case (dashed line) and the free vibration case (continuous line)

This expression coincides with the one obtained by Zhang et al. [48]. When the number of elements goes to infinity, we get following result

$$
\lim _{n_{y} \rightarrow \infty} \frac{1}{e_{0}^{2}}=12\left\{\frac{\alpha^{2} m^{2}\left(\alpha^{2} m^{2}+1\right)(s+1)+\alpha^{6} m^{6}+\alpha^{4} m^{4}+\alpha^{2} m^{2} s+s}{\alpha^{6} m^{6}+2 \alpha^{4} m^{4} s-\alpha^{4} m^{4}-\alpha^{2} m^{2} s+2 \alpha^{2} m^{2}+s}\right\}
$$

It can be seen that Eq. (172) coincides with the results of Challamel et al. [20]. The small length scale coefficient depends on $m$, the buckling mode and $\alpha$, the aspect ratio. Considering a uniaxial load $(s=0)$, the expression of the small length scale coefficient is compared between the different models (see Figs. 5 and 6).

The small length scale coefficient is now performed in the case of free transverse vibration $(s=0, N=0)$. In a similar manner to the beam models, the small length scale coefficient is obtained by equating the vibration frequency in Eqs. (115) and (170), with the assumption of one-half wave in the $y$-direction,

$$
\frac{1}{e_{0}^{2}}=\frac{4 \pi^{2}\left[(\alpha m)^{2}+1\right] n_{y}^{4}\left(\cos \frac{\alpha m \pi}{n_{y}}+\cos \frac{\pi}{n_{y}}-2\right)^{2}}{n_{y}^{2} \pi^{4}\left(1+m^{2} \alpha^{2}\right)^{2}-4 n_{y}^{6}\left(\cos \frac{\alpha m \pi}{n_{y}}+\cos \frac{\pi}{n_{y}}-2\right)^{2}}
$$

When the number of elements goes to infinity, we obtain:

$$
\lim _{n_{y} \rightarrow \infty} \frac{1}{e_{0}}=\sqrt{6} \sqrt{\frac{\left(m^{2} \alpha^{2}+1\right)^{2}}{1+m^{4} \alpha^{4}}}
$$


Table 1 Mean calibrated small length scale coefficient considering two continualized models and two phenomenological models for beams and plates

\begin{tabular}{|c|c|c|c|c|c|c|c|c|}
\hline \multirow[t]{3}{*}{ Order } & \multicolumn{4}{|c|}{ Beams } & \multicolumn{4}{|l|}{ Plates } \\
\hline & \multicolumn{2}{|c|}{ Continualized } & \multicolumn{2}{|c|}{ Phenomenological } & \multicolumn{2}{|c|}{ Continualized } & \multicolumn{2}{|c|}{ Phenomenological } \\
\hline & 4 & 6 & 4 & 6 & 4 & 6 & 4 & 6 \\
\hline Statics & 0.288 & 0.288 & 0.288 & 0.204 & 0.288 & 0.288 & $0.191-0.408$ & $0.182-0.288$ \\
\hline Free vibrations & 0.288 & 0.288 & 0.408 & 0.288 & 0.288 & 0.288 & $0.288-0.408$ & 0.288 \\
\hline Gap (\%) & 0 & 0 & 50 & 29.2 & 0 & 0 & 9.6 & 10.4 \\
\hline
\end{tabular}

These results coincide with those of Zhang et al. $[18,48]$. The small length scale coefficient is not constant: it is structural problem dependent. The expression of the small length scale coefficient is plotted for each model. Considering the Eringen's model, the small length scale coefficient lies between $1 / \sqrt{12}$ and $1 / \sqrt{6}$, and it varies with respect to different mode numbers and aspect ratios. As illustrated in Fig. 8b, it depends on the load, which contradicts the commonly made assumption of a constant length scale.

For $m=1$ and $\mathrm{n}=1$, the small length scale coefficient is obtained for various values of the aspect ratio $\alpha$ in the case of free vibration and statics (see Fig. 5; Table 1). Whereas for the continualized models, it is constant in both situations, the traditional fourth-order phenomenological model provides two small length scales coefficients in statics and in dynamics and in both situations, it depends on the aspect ratio $\alpha$. The gap between the static and dynamic case increases with the vibration mode and the aspect ratio $\alpha$ and reaches $29.2 \%$ for a square plate $(\alpha=1)$.

Furthermore, in order to compare the models, the calibrated small length scale coefficients are reported in Table 1, considering plates and beams. A significant difference is noticed for the fourth-order phenomenological model in statics and in free vibration problems.

\subsection{A sixth-order phenomenological approach}

As for a beam, an attempt is made to develop a model comprising the gradient elasticity and nonlocal elasticity models. Recently, Challamel et al. [20] suggested the following moment-curvature relations:

$$
\begin{gathered}
{\left[1-\left(e_{0} a\right)^{2} \nabla^{2}\right] M_{x x}=D\left[\frac{\partial^{2} w}{\partial x^{2}}+\nu \frac{\partial^{2} w}{\partial y^{2}}-\left(e_{0} a\right)^{2}\left(\frac{\partial^{4} w}{\partial x^{2} \partial y^{2}}-\frac{\partial^{4} w}{\partial x^{4}}\right)\right]} \\
{\left[1-\left(e_{0} a\right)^{2} \nabla^{2}\right] M_{y y}=D\left[\frac{\partial^{2} w}{\partial y^{2}}+\nu \frac{\partial^{2} w}{\partial x^{2}}-\left(e_{0} a\right)^{2}\left(\frac{\partial^{4} w}{\partial x^{2} \partial y^{2}}-\frac{\partial^{4} w}{\partial y^{4}}\right)\right]} \\
{\left[1-\left(e_{0} a\right)^{2} \nabla^{2}\right] M_{x y}=D(1-v) \frac{\partial^{2} w}{\partial x \partial y}}
\end{gathered}
$$

This model is close to the classical nonlocal elasticity Eringen model. Indeed, the left-hand sides of Eqs. (175)(177) are the same as those in the Eringen's model but the right hand side includes an additional term.

By substituting Eqs. (175)-(177) into Eq. (104), multiplying by $\left[1-\left(e_{0} a\right)^{2}\left(\partial^{2} / \partial x^{2}+\partial^{2} / \partial y^{2}\right)\right]$, we get

$$
\begin{aligned}
& D\left[\frac{\partial^{4} w}{\partial x^{4}}+2 \frac{\partial^{4} w}{\partial x^{2} \partial y^{2}}+\frac{\partial^{4} w}{\partial y^{4}}+\left(e_{0} a\right)^{2}\left(\frac{\partial^{6} w}{\partial x^{6}}-\frac{\partial^{6} w}{\partial x^{4} \partial y^{2}}-\frac{\partial^{6} w}{\partial x^{2} \partial y^{4}}+\frac{\partial^{6} w}{\partial y^{6}}\right)\right] \\
& -m_{0} \omega^{2}\left[w+\left(e_{0} a\right)^{2}\left(\frac{\partial^{2} w}{\partial x^{2}}+\frac{\partial^{2} w}{\partial y^{2}}\right)\right]+P\left(\frac{\partial^{2} w}{\partial x^{2}}+s \frac{\partial^{2} w}{\partial y^{2}}\right) \\
& -\left(e_{0} a\right)^{2} P(1+s)\left(\frac{\partial^{4} w}{\partial x^{2} \partial y^{2}}\right)-\left(e_{0} a\right)^{2} P\left(\frac{\partial^{4} w}{\partial x^{4}}+s \frac{\partial^{4} w}{\partial y^{4}}\right)=0
\end{aligned}
$$

In contrast to the fourth-order phenomenological and nonlocal Eringen's model, we obtain a sixth-order space derivative equation. This equation is different from the equation obtained in the sixth-order continualized model. Thus, as for the sixth-order continualized model, it requires two additional boundary conditions. 
Equation (178) may be also obtained from $U, T$ and $W$ given by Eqs. (150), (151) and (164), respectively:

$$
\begin{aligned}
U(w)= & \frac{1}{2} \iint_{\mathfrak{D}} D\left[\left(\frac{\partial^{2} w}{\partial x^{2}}\right)^{2}+2 \nu \frac{\partial^{2} w}{\partial x^{2}} \frac{\partial^{2} w}{\partial y^{2}}+\left(\frac{\partial^{2} w}{\partial y^{2}}\right)^{2}+2(1-\nu)\left(\frac{\partial^{2} w}{\partial x \partial y}\right)^{2}\right. \\
& -\left(e_{0} a\right)^{2}\left(\left(\frac{\partial^{3} w}{\partial x^{3}}\right)^{2}+\left(\frac{\partial^{3} w}{\partial y^{3}}\right)^{2}\right) \\
& \left.+\left(e_{0} a\right)^{2}\left(\left(\frac{\partial^{3} w}{\partial x^{2} \partial y}\right)^{2}+\left(\frac{\partial^{3} w}{\partial x \partial y^{2}}\right)^{2}\right)\right] \mathrm{d} x \mathrm{~d} y \\
T(w)= & \frac{1}{2} \iint_{\mathfrak{D}} m_{0} \omega^{2}\left[w^{2}+\left(e_{0} a\right)^{2}\left(\left(\frac{\partial w}{\partial x}\right)^{2}+\left(\frac{\partial w}{\partial y}\right)^{2}\right)\right] \mathrm{d} x \mathrm{~d} y \\
W(w)= & \frac{1}{2} \iint_{\mathfrak{D}} P\left[\left(\frac{\partial w}{\partial x}\right)^{2}+s\left(\frac{\partial w}{\partial y}\right)^{2}+\left(e_{0} a\right)^{2}\left(\frac{\partial^{2} w}{\partial x^{2}}\right)^{2}+\left(e_{0} a\right)^{2} s\left(\frac{\partial^{2} w}{\partial y^{2}}\right)^{2}\right. \\
& \left.+\left(e_{0} a\right)^{2}(s+1) \frac{\partial^{2} w}{\partial x^{2}} \frac{\partial^{2} w}{\partial y^{2}}\right] \mathrm{d} x \mathrm{~d} y
\end{aligned}
$$

Thus, by applying the Hamilton principle, we get Eq. (178) with $I$ given in (137) defined as follows:

$$
\begin{aligned}
I(x, y, t)= & \left(e_{0} a\right)^{2} D\left[\frac{\partial^{3} w}{\partial x \partial y^{2}} \frac{\partial^{2} \delta w}{\partial y^{2}}-\frac{\partial^{3} w}{\partial x^{3}} \frac{\partial^{2} \delta w}{\partial x^{2}}\right] d y+\left(e_{0} a\right)^{2} D\left[\frac{\partial^{3} w}{\partial x^{2} \partial y} \frac{\partial^{2} \delta w}{\partial x^{2}}-\frac{\partial^{3} w}{\partial y^{3}} \frac{\partial^{2} \delta w}{\partial y^{2}}\right] \mathrm{d} y \\
& +\left\{D\left(v \frac{\partial^{2} w}{\partial x^{2}}+\frac{\partial^{2} w}{\partial y^{2}}\right)-D\left(e_{0} a\right)^{2}\left(\frac{\partial^{4} w}{\partial x^{2} \partial y^{2}}-\frac{\partial^{4} w}{\partial y^{4}}\right)\right. \\
& \left.-\frac{\left(e_{0} a\right)^{2} P}{2}\left[2 s \frac{\partial^{2} w}{\partial y^{2}}+(1+s) \frac{\partial^{2} w}{\partial x^{2}}\right]\right\} \delta\left(\frac{\partial w}{\partial y}\right) \mathrm{d} x \\
& +\left\{D\left(\frac{\partial^{2} w}{\partial x^{2}}+v \frac{\partial^{2} w}{\partial y^{2}}\right)-D\left(e_{0} a\right)^{2}\left(\frac{\partial^{4} w}{\partial x^{2} \partial y^{2}}-\frac{\partial^{4} w}{\partial x^{4}}\right)\right. \\
& \left.-\frac{\left(e_{0} a\right)^{2} P}{2}\left[2 \frac{\partial^{2} w}{\partial x^{2}}+(1+s) \frac{\partial^{2} w}{\partial y^{2}}\right]\right\} \delta\left(\frac{\partial w}{\partial x}\right) \mathrm{d} y \\
& -\left\{D\left[\frac{\partial^{3} w}{\partial x^{3}}+\frac{\partial^{3} w}{\partial x \partial y^{2}}+\left(e_{0} a\right)^{2}\left(\frac{\partial^{5} w}{\partial x^{5}}-\frac{\partial^{5} w}{\partial x^{3} \partial y^{2}}\right)\right]+P \frac{\partial w}{\partial x}\right. \\
& \left.+\frac{\left(e_{0} a\right)^{2} P}{2}\left[2 \frac{\partial^{3} w}{\partial x^{3}}+(1+s) \frac{\partial^{3} w}{\partial x \partial y^{2}}\right]+\left(e_{0} a\right)^{2} m_{0} \omega^{2} \frac{\partial w}{\partial x}\right\} \delta w \mathrm{~d} y \\
& -\left\{D\left[\frac{\partial^{3} w}{\partial y^{3}}+\frac{\partial^{3} w}{\partial x^{2} \partial y}+\left(e_{0} a\right)^{2}\left(\frac{\partial^{5} w}{\partial y^{5}}-\frac{\partial^{5} w}{\partial x^{2} \partial y^{3}}\right)\right]+s P \frac{\partial w}{\partial y}\right. \\
& \left.+\frac{\left(e_{0} a\right)^{2} P}{2}\left[2 s \frac{\partial^{3} w}{\partial y^{3}}+(1+s) \frac{\partial^{3} w}{\partial x^{2} \partial y}\right]+\left(e_{0} a\right)^{2} m_{0} \omega^{2} \frac{\partial w}{\partial y}\right\} \delta w \mathrm{~d} x
\end{aligned}
$$

In view of boundary conditions, substituting Eq. (139) in Eq. (178), the solution is provided:

$$
\begin{aligned}
\Omega^{2}= & \frac{\left(\frac{\alpha L}{a}\right)^{2}\left(n^{2}+m^{2} \alpha^{2}\right)^{2} \pi^{4}-e_{0}^{2} \pi^{6}\left[(\alpha m)^{6}-(\alpha m)^{4} n^{2}-(\alpha m)^{2} n^{4}+n^{6}\right]}{\left[\left(\frac{\alpha L}{a}\right)^{2}+e_{0}^{2} \pi^{2}\left[(\alpha m)^{2}+n^{2}\right]\right]} \\
& -\lambda \pi^{2} \frac{\left[e_{0}^{2} \pi^{2}(\alpha m n)^{2}(1+s)+e_{0}^{2} \pi^{2}\left[(\alpha m)^{4}+s n^{4}\right]+\left(\frac{\alpha L}{a}\right)^{2}\left[(\alpha m)^{2}+s n^{2}\right]\right]}{\left[\left(\frac{\alpha L}{a}\right)^{2}+e_{0}^{2} \pi^{2}\left[(\alpha m)^{2}+n^{2}\right]\right]}
\end{aligned}
$$


In the static case, $\Omega^{2}=0$, assuming $=1$, the buckling load parameter is

$$
\lambda=\min _{(m, n)} \pi^{4}\left\{\frac{\left(\frac{\alpha L}{a}\right)^{2}\left(1+m^{2} \alpha^{2}\right)^{2}-e_{0}^{2} \pi^{2}\left[(\alpha m)^{6}-(\alpha m)^{4}-(\alpha m)^{2}+1\right]}{\left[e_{0}^{2} \pi^{2}(\alpha m)^{2}(1+s)+e_{0}^{2} \pi^{2}\left[(\alpha m)^{4}+s\right]+\left(\frac{\alpha L}{a}\right)^{2}\left[(\alpha m)^{2}+s\right]\right]}\right\}
$$

For the particular case of a uniaxial load, i.e. $s=0$, the squared natural frequency parameter becomes:

$$
\begin{aligned}
\Omega^{2}= & \frac{\left(\frac{\alpha L}{a}\right)^{2}\left(n^{2}+m^{2} \alpha^{2}\right)^{2} \pi^{4}-e_{0}^{2} \pi^{6}\left[(\alpha m)^{6}-(\alpha m)^{4} n^{2}-(\alpha m)^{2} n^{4}+n^{6}\right]}{\left[\left(\frac{\alpha L}{a}\right)^{2}+e_{0}^{2} \pi^{2}\left[(\alpha m)^{2}+n^{2}\right]\right]} \\
& -\lambda \pi^{2}(\alpha m)^{2} \frac{e_{0}^{2} \pi^{2} n^{2}+e_{0}^{2} \pi^{2}\left[(\alpha m)^{2}\right]+\left(\frac{\alpha L}{a}\right)^{2}}{\left[\left(\frac{\alpha L}{a}\right)^{2}+e_{0}^{2} \pi^{2}\left[(\alpha m)^{2}+n^{2}\right]\right]}
\end{aligned}
$$

Furthermore, in free vibration, the frequency is calculated from $\lambda=0$ in Eq. (185)

$$
\Omega^{2}=\frac{\left(\frac{\alpha L}{a}\right)^{2}\left(n^{2}+m^{2} \alpha^{2}\right)^{2} \pi^{4}}{\left[\left(\frac{\alpha L}{a}\right)^{2}+e_{0}^{2} \pi^{2}\left[(\alpha m)^{2}+n^{2}\right]\right]}-e_{0}^{2} \pi^{6} \frac{\left[(\alpha m)^{6}-(\alpha m)^{4} n^{2}-(\alpha m)^{2} n^{4}+n^{6}\right]}{\left[\left(\frac{\alpha L}{a}\right)^{2}+e_{0}^{2} \pi^{2}\left[(\alpha m)^{2}+n^{2}\right]\right]}
$$

As for the fourth-order continualized model, the small length scale coefficient has to be calibrated. Thus, by equating the buckling load from Eqs. (116) and (184), the small length scale coefficient is

$$
\frac{1}{e_{0}^{2}}=\frac{\frac{2 n_{y}^{2}\left(\cos \frac{\alpha m \pi}{n_{y}}+\cos \frac{\pi}{n_{y}}-2\right)^{2}}{1+s-\cos \frac{\alpha m \pi}{n_{y}}-s \cos \frac{\pi}{n_{y}}} \pi^{2}\left[(\alpha m)^{2}(1+s)+\left[(\alpha m)^{4}+s\right]\right]+\pi^{4}\left[(\alpha m)^{6}-(\alpha m)^{4}-(\alpha m)^{2}+1\right]}{n_{y}^{2} \pi^{2}\left(1+m^{2} \alpha^{2}\right)^{2}-\frac{2 n_{y}^{4}\left(\cos \frac{\alpha m \pi}{\left.n_{y}+\cos \frac{\pi}{n_{y}}-2\right)^{2}}\right.}{1+s-\cos \frac{\alpha m \pi}{n_{y}}-s \cos \frac{\pi}{n_{y}}}\left[(\alpha m)^{2}+s\right]}
$$

When the number of elements goes to infinity, it yields

$$
\lim _{n_{y} \rightarrow \infty} \frac{1}{e_{0}^{2}}=24\left\{\frac{\alpha^{6} m^{6}+\alpha^{4} m^{4} s+\alpha^{2} m^{2}+s}{\alpha^{6} m^{6}+2 \alpha^{4} m^{4} s-\alpha^{4} m^{4}-\alpha^{2} m^{2} s+2 \alpha^{2} m^{2}+s}\right\}
$$

Again, the small length scale coefficient is structural dependent: it varies with the buckling mode and the aspect ratio. Considering a uniaxial load $(s=0)$, the expression of the small length scale coefficient is compared between the different models (see Figs. 5, 6). The gap between the value in statics in dynamics and statics increases with respect to the aspect ratio $\alpha$.

Furthermore, the calibrated small length scale coefficient is obtained by equating the vibration frequency in Eqs. (115) and (186), in the case of free transverse vibration $(s=0, N=0)$.,

$$
\frac{1}{e_{0}^{2}}=\frac{4 \pi^{2}\left[(\alpha m)^{2}+1\right] n_{y}^{4}\left(\cos \frac{\alpha m \pi}{n_{y}}+\cos \frac{\pi}{n_{y}}-2\right)^{2}+\pi^{6}\left[(\alpha m)^{6}-(\alpha m)^{4}-(\alpha m)^{2}+1\right]}{n_{y}^{2} \pi^{4}\left(1+m^{2} \alpha^{2}\right)^{2}-4 n_{y}^{6}\left(\cos \frac{\alpha m \pi}{n_{y}}+\cos \frac{\pi}{n_{y}}-2\right)^{2}}
$$

When the number of elements goes to infinity, it yields:

$$
\lim _{n_{y} \rightarrow \infty} e_{0}=\frac{1}{\sqrt{12}}
$$

In this particular case, the small length scale coefficient is constant equal to $e_{0}^{2}=1 / 12$, same value than for the continualized models, and so, is structural independent. This result is much better than for the fourth-order continualized model. The expression of the small length scale coefficient is plotted for each model (see Fig. 6). 


\subsection{General solutions}

\subsubsection{Buckling load and natural frequency}

The four versions of governing differential equation can be written in a canonical form as

$$
\begin{aligned}
& D\left[\frac{\partial^{4} w}{\partial x^{4}}+2 \frac{\partial^{4} w}{\partial x^{2} \partial y^{2}}+\frac{\partial^{4} w}{\partial y^{4}}+\gamma_{1}\left(e_{0} a\right)^{2}\left(\frac{\partial^{6} w}{\partial x^{6}}+\frac{\partial^{6} w}{\partial y^{6}}\right)-\left(\gamma_{1}+4 \gamma_{3}\right)\left(e_{0} a\right)^{2}\left(\frac{\partial^{6} w}{\partial x^{4} \partial y^{2}}+\frac{\partial^{6} w}{\partial x^{2} \partial y^{4}}\right)\right] \\
& -m_{0} \omega^{2} w+\left(1+\gamma_{3}\right)\left(e_{0} a\right)^{2} m_{0} \omega^{2}\left(\frac{\partial^{2} w}{\partial x^{2}}+\frac{\partial^{2} w}{\partial y^{2}}\right)+P\left(\frac{\partial^{2} w}{\partial x^{2}}+s \frac{\partial^{2} w}{\partial y^{2}}\right) \\
& -\left(1+\gamma_{3}\right)\left(e_{0} a\right)^{2} P(1+s) \frac{\partial^{4} w}{\partial x^{2} \partial y^{2}}-\left(\gamma_{2}+\gamma_{3}\right)\left(e_{0} a\right)^{2} P\left(\frac{\partial^{4} w}{\partial x^{4}}+s \frac{\partial^{4} w}{\partial y^{4}}\right)=0
\end{aligned}
$$

where three control scalar parameters, namely $\gamma_{1}, \gamma_{2}$ and $\gamma_{3}$ are introduced with $\left(\gamma_{1}, \gamma_{2}, \gamma_{3}\right)$ is equal to $(0,0,1)$, $(1,0,0),(0,1,0)$ and $(1,1,0)$ for the fourth-order continualized model, the sixth-order order continualized model, the fourth-order phenomenological model and the sixth-order phenomenological model, respectively. The results coincide with the ones of Challamel et al. [20] for $\gamma_{3}=0$, i.e. for the nonlocal plate models considered except for the fourth-order continualized nonlocal plate model which is introduced herein.

The substitution of Eq. (139) into Eq. (191) furnishes the following solution

$$
\begin{aligned}
\Omega^{2}= & \frac{\left(\frac{\alpha L}{a}\right)^{2}\left(n^{2}+m^{2} \alpha^{2}\right)^{2} \pi^{4}}{\left[\left(\frac{\alpha L}{a}\right)^{2}+\left(1+\gamma_{3}\right) e_{0}^{2} \pi^{2}\left[(\alpha m)^{2}+n^{2}\right]\right]}-\gamma_{1} e_{0}^{2} \pi^{6} \frac{\left[(\alpha m)^{6}-(\alpha m)^{4} n^{2}-(\alpha m)^{2} n^{4}+n^{6}\right]}{\left[\left(\frac{\alpha L}{a}\right)^{2}+\left(1+\gamma_{3}\right) e_{0}^{2} \pi^{2}\left[(\alpha m)^{2}+n^{2}\right]\right]} \\
& +4 \gamma_{3} e_{0}^{2} \pi^{6}(\alpha m)^{2} n^{2} \frac{\left[(\alpha m)^{2}+n^{2}\right]}{\left[\left(\frac{\alpha L}{a}\right)^{2}+\left(1+\gamma_{3}\right) e_{0}^{2} \pi^{2}\left[(\alpha m)^{2}+n^{2}\right]\right]} \\
& -\lambda \pi^{2} \frac{\left[e_{0}^{2} \pi^{2}(\alpha m n)^{2}(1+s)\left(1+\gamma_{3}\right)+e_{0}^{2} \pi^{2}\left(\gamma_{2}+\gamma_{3}\right)\left[(\alpha m)^{4}+s n^{4}\right]+\left(\frac{\alpha L}{a}\right)^{2}\left[(\alpha m)^{2}+s n^{2}\right]\right]}{\left[\left(\frac{\alpha L}{a}\right)^{2}+\left(1+\gamma_{3}\right) e_{0}^{2} \pi^{2}\left[(\alpha m)^{2}+n^{2}\right]\right]}
\end{aligned}
$$

Remarkably, when there is no load $(N=0)$, the vibration frequency following the continualized model based on the Padé approximant and the hybrid continualized model turns out to be equal.

When $s=0$, the squared natural frequency parameter is:

$$
\begin{aligned}
\Omega^{2}= & \frac{\left(\frac{\alpha L}{a}\right)^{2}\left(n^{2}+m^{2} \alpha^{2}\right)^{2} \pi^{4}}{\left[\left(\frac{\alpha L}{a}\right)^{2}+\left(1+\gamma_{3}\right) e_{0}^{2} \pi^{2}\left[(\alpha m)^{2}+n^{2}\right]\right]} \\
& -\lambda \pi^{2}(\alpha m)^{2} \frac{e_{0}^{2} \pi^{2} n^{2}\left(1+\gamma_{3}\right)+e_{0}^{2} \pi^{2}\left(\gamma_{2}+\gamma_{3}\right)\left[(\alpha m)^{2}\right]+\left(\frac{\alpha L}{a}\right)^{2}}{\left[\left(\frac{\alpha L}{a}\right)^{2}+\left(1+\gamma_{3}\right) e_{0}^{2} \pi^{2}\left[(\alpha m)^{2}+n^{2}\right]\right]} \\
& -\gamma_{1} e_{0}^{2} \pi^{6} \frac{\left[(\alpha m)^{6}-(\alpha m)^{4} n^{2}-(\alpha m)^{2} n^{4}+n^{6}\right]}{\left[\left(\frac{\alpha L}{a}\right)^{2}+\left(1+\gamma_{3}\right) e_{0}^{2} \pi^{2}\left[(\alpha m)^{2}+n^{2}\right]\right]} \\
& +4 \gamma_{3} e_{0}^{2} \pi^{6}(\alpha m)^{2} n^{2} \frac{\left[(\alpha m)^{2}+n^{2}\right]}{\left[\left(\frac{\alpha L}{a}\right)^{2}+\left(1+\gamma_{3}\right) e_{0}^{2} \pi^{2}\left[(\alpha m)^{2}+n^{2}\right]\right]}
\end{aligned}
$$

Furthermore, in free vibration, the frequency is calculated from $\lambda=0$ in Eq. (192) 


$$
\begin{aligned}
\Omega^{2}= & \frac{\left(\frac{\alpha L}{a}\right)^{2}\left(n^{2}+m^{2} \alpha^{2}\right)^{2} \pi^{4}}{\left[\left(\frac{\alpha L}{a}\right)^{2}+\left(1+\gamma_{3}\right) e_{0}^{2} \pi^{2}\left[(\alpha m)^{2}+n^{2}\right]\right]}-\gamma_{1} e_{0}^{2} \pi^{6} \frac{\left[(\alpha m)^{6}-(\alpha m)^{4} n^{2}-(\alpha m)^{2} n^{4}+n^{6}\right]}{\left[\left(\frac{\alpha L}{a}\right)^{2}+\left(1+\gamma_{3}\right) e_{0}^{2} \pi^{2}\left[(\alpha m)^{2}+n^{2}\right]\right]} \\
& +4 \gamma_{3} e_{0}^{2} \pi^{6}(\alpha m)^{2} n^{2} \frac{\left[(\alpha m)^{2}+n^{2}\right]}{\left[\left(\frac{\alpha L}{a}\right)^{2}+\left(1+\gamma_{3}\right) e_{0}^{2} \pi^{2}\left[(\alpha m)^{2}+n^{2}\right]\right]}
\end{aligned}
$$

From the previous expressions of the small length scale coefficients, several observations can be made. The small length scale coefficient turns out to be not a constant. It depends on the initial load $(N$ or $\lambda)$, the vibration frequency $\Omega$, the vibration/buckling mode $(m, n)$, the material (the internal characteristic $a$ ) and the geometry (aspect ratio $\alpha$ and one of the length of the beam $L$ ) [20]. Thus, it is calibrated by equating the buckling stress from the discrete model and the continuous one:

$$
\begin{gathered}
\frac{1}{e_{0}^{2}}=\frac{\frac{2 n_{y}^{2}\left(\cos \frac{\alpha m \pi}{n_{y}}+\cos \frac{\pi}{n_{y}}-2\right)^{2}}{1+s-\cos \frac{\alpha m \pi}{n_{y}}-s \cos \frac{\pi}{n_{y}}} \pi^{2}\left[(\alpha m)^{2}(1+s)\left(1+\gamma_{3}\right)+\left(\gamma_{2}+\gamma_{3}\right)\left[(\alpha m)^{4}+s\right]\right]}{n_{y}^{2} \pi^{2}\left(1+m^{2} \alpha^{2}\right)^{2}-\frac{2 n_{y}^{4}\left(\cos \frac{\alpha m \pi}{n_{y}}+\cos \frac{\pi}{n_{y}}-2\right)^{2}}{1+s-\cos \frac{\alpha m \pi}{n_{y}}-s \cos \frac{\pi}{n_{y}}}\left[(\alpha m)^{2}+s\right]} \\
+\frac{\gamma_{1} \pi^{4}\left[(\alpha m)^{6}-(\alpha m)^{4}-(\alpha m)^{2}+1\right]-4 \gamma_{3} \pi^{4}(\alpha m)^{2}\left[(\alpha m)^{2}+1\right]}{n_{y}^{2} \pi^{2}\left(1+m^{2} \alpha^{2}\right)^{2}-\frac{2 n_{y}^{4}\left(\cos \frac{\alpha m \pi}{n_{y}}+\cos \frac{\pi}{n_{y}}-2\right)^{2}}{1+s-\cos \frac{\alpha m \pi}{n_{y}}-s \cos \frac{\pi}{n_{y}}}\left[(\alpha m)^{2}+s\right]}
\end{gathered}
$$

When the number of elements goes to infinity, we get following result

$$
\begin{aligned}
\lim _{n_{y} \rightarrow \infty} \frac{1}{e_{0}^{2}}= & 12\left\{\frac{\alpha^{2} m^{2}\left(\alpha^{2} m^{2}+1\right)(s+1)}{\alpha^{6} m^{6}+2 \alpha^{4} m^{4} s-\alpha^{4} m^{4}-\alpha^{2} m^{2} s+2 \alpha^{2} m^{2}+s}\right. \\
& +\left(\gamma_{1}+\gamma_{3}\right) \frac{\alpha^{6} m^{6}+\alpha^{4} m^{4} s-2 \alpha^{4} m^{4}-2 \alpha^{2} m^{2} s+\alpha^{2} m^{2}+s}{\alpha^{6} m^{6}+2 \alpha^{4} m^{4} s-\alpha^{4} m^{4}-\alpha^{2} m^{2} s+2 \alpha^{2} m^{2}+s} \\
& \left.+\gamma_{2} \frac{\alpha^{6} m^{6}+\alpha^{4} m^{4}+\alpha^{2} m^{2} s+s}{\alpha^{6} m^{6}+2 \alpha^{4} m^{4} s-\alpha^{4} m^{4}-\alpha^{2} m^{2} s+2 \alpha^{2} m^{2}+s}\right\}
\end{aligned}
$$

For the nonlocal Eringen's model and the sixth-order continualized models, Eq. (196) coincides with the results of Challamel et al. [20]. It can be checked from Eq. (196) that the small length scale coefficient is constant for the fourth-order and sixth-order continualized models, i.e. for $\gamma_{1}+\gamma_{3}=1$ and $\gamma_{2}=0$, and is equal to $e_{0}^{2}=1 / 12$. In contrast to the nonlocal Eringen's model and the phenomenological models, it is independent of $m$, the buckling mode and $\alpha$, the aspect ratio. Considering a uniaxial load $(s=0)$, the expression of the small length scale coefficient is compared between the different models (see Figs. 5, 6).

The small length scale coefficient is now performed in the case of free transverse vibration $(s=0, N=0)$. In a similar manner to the beam models, the small length scale coefficient is obtained by equating the vibration frequency in Eqs. (115) and (194), with the assumption of one-half wave in the $y$-direction,

$$
\begin{aligned}
\frac{1}{e_{0}^{2}}= & \frac{4 \pi^{2}\left[(\alpha m)^{2}+1\right] n_{y}^{4}\left(\cos \frac{\alpha m \pi}{n_{y}}+\cos \frac{\pi}{n_{y}}-2\right)^{2}}{n_{y}^{2} \pi^{4}\left(1+m^{2} \alpha^{2}\right)^{2}-4 n_{y}^{6}\left(\cos \frac{\alpha m \pi}{n_{y}}+\cos \frac{\pi}{n_{y}}-2\right)^{2}} \\
& +\gamma_{1} \frac{\pi^{6}\left[(\alpha m)^{6}-(\alpha m)^{4}-(\alpha m)^{2}+1\right]}{n_{y}^{2} \pi^{4}\left(1+m^{2} \alpha^{2}\right)^{2}-4 n_{y}^{6}\left(\cos \frac{\alpha m \pi}{n_{y}}+\cos \frac{\pi}{n_{y}}-2\right)^{2}} \\
& +\gamma_{3} \frac{4 \pi^{2}\left[(\alpha m)^{2}+1\right] n_{y}^{4}\left(\cos \frac{\alpha m \pi}{n_{y}}+\cos \frac{\pi}{n_{y}}-2\right)^{2}-4 \pi^{6}(\alpha m)^{2}\left[(\alpha m)^{2}+1\right]}{n_{y}^{2} \pi^{4}\left(1+m^{2} \alpha^{2}\right)^{2}-4 n_{y}^{6}\left(\cos \frac{\alpha m \pi}{n_{y}}+\cos \frac{\pi}{n_{y}}-2\right)^{2}}
\end{aligned}
$$

When the number of elements goes to infinity, we obtain:

$$
\lim _{n_{y} \rightarrow \infty} \frac{1}{e_{0}}=\sqrt{6} \sqrt{\frac{\left(m^{2} \alpha^{2}+1\right)^{2}}{1+m^{4} \alpha^{4}}+\left(\gamma_{1}+\gamma_{3}\right) \frac{\left(m^{2} \alpha^{2}-1\right)^{2}}{1+m^{4} \alpha^{4}}}
$$


It can be checked from Eq. (198) that the small length scale coefficient is constant for the continualized nonlocal models, i.e. for $\gamma_{1}+\gamma_{3}=1$ and $\gamma_{2}=0$, and is equal to $e_{0}^{2}=1 / 12$, both for buckling and vibration analyses.

The expression of the small length scale coefficient is plotted for each model. As explained above, considering the Eringen's model, the small length scale coefficient depends on the load, which contradicts the commonly made assumption of a constant length-scale.

Let us compare the small length scale coefficient with $m=1$ and $\mathrm{n}=1$ in the case of free vibration and statics for different values of the aspect ratio $\alpha$ (see Fig. 5). For the continualized models, it is constant for both cases. For the phenomenological models, a gap occurs between the two between the two situations. This gap increases in both cases with the aspect ratio $\alpha$. When the vibration mode increases, a gap between the Eringen and the microstructured beam-grid model occurs. This gap increases with the vibration mode, whereas for the model based on the Padé's approximant, the curve matches exactly the exact solution.

The traditional nonlocal Eringen's model provides two small length scales coefficients in statics and in dynamics and in both situations, it depends on the aspect ratio $\alpha$. The sixth-order phenomenological model gives a constant small length scale coefficient in free vibration but when an initial load is taken into account, the calibrated coefficient depends on the aspect ratio. Considering the continualized models, by definition, the small length scale coefficient has the same value in statics and in free vibrations. Thus, the continualized models are much better than the phenomenological models based on postulated nonlocal constitutive laws.

As it can be seen in Table 1, based on the microstructured model, the continualized models provide better results than the phenomenoplogical models, based on postulated nonlocal constitutive laws. Thus, assuming that the best calibration of the small length scale coefficient is obtained through the first continualized model and so, is equal to 0.288 , the traditional fourth-order phenomenological model (nonlocal Eringen's theory) overestimates its value (gap of 7.7\%), whereas the sixth-order phenomenological model underestimates the value (gap of 9.6\%). Such differences cannot be ignored, and it appears that the traditional model is not convenient for the study of vibrations of plates.

Furthermore, in order to compare the models, the calibrated small length scale coefficients are reported in Table 1 considering plates and beams. The results are more disparate for plates than for beams. The continualized models are based on the lattice model. Thus, the calibrated small length scale coefficients are the same in statics (calibration from the nondimensional buckling load) and in dynamics (calibration from the nondimensional natural frequency). They are naturally structural problem independent. The sixth-order space derivative models require the introduction of sixth boundary conditions and consequently, could be considered unnecessary compared to the fourth-order space derivative models. However, these models lead to a nearly constant small length scale coefficient, independent of the load. Thus, this establishes the superiority of such models.

Furthermore, we define $\Delta e_{0}$ the gap between the small length scale coefficient, calibrated in the fourthorder and sixth-order phenomenological model and the value of the coefficient following the continualized models $\left(e_{0}=1 / \sqrt{12}\right)$ :

$$
\Delta e_{0}=\frac{e_{0}-1 / \sqrt{12}}{1 / \sqrt{12}}
$$

The value of $\Delta e_{0}$ is plotted in Figs. 6 and 7. It can be seen that the sixth-order phenomenological model, in the case of free vibration, is constant and coincides with the exact value obtained through the continualized models. Considering the fourth-order phenomenological model, in free vibration, the gap decreases with $\alpha$. In statics, it decreases initially and then it increases.

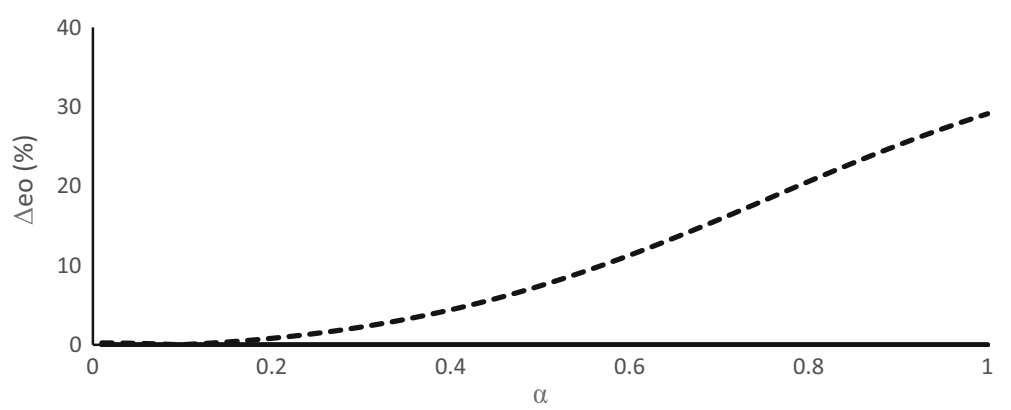

Fig. 7 Gap between the small length scale coefficient calibrated in the sixth-order phenomenological model and the exact one obtained in the continualized model in the static case (dashed line) and the free vibration case (continuous line) 


\section{Conclusion}

In this study, four nonlocal beam and plate models have been discussed: two are continualized lattice-based models and two other phenomenological models are based on postulated nonlocal constitutive laws. As already investigated in different papers, the calibrated small length scale coefficient can take different values considering the phenomenological models for static or dynamics analyses. Thus, it turns out to be dependent on the load and the structural problem. Although they do not preserve the locality of the balance equations, the continualized models have the advantage that the small length scale coefficient has the same value in buckling and in vibration.

While the Eringen's derivation for beams and plates lead to a fourth-order space derivative differential equation, some of the equations developed herein possess sixth-order space derivatives. The additional higherorder boundary conditions may be obtained through variational principles as detailed in the paper. The fourthorder phenomenological model does not require additional boundary conditions and, consequently, is simpler than the sixth-order continualized models and the sixth-order phenomenological model.

Furthermore, by using phenomenological nonlocal models such as Eringen's nonlocal differential model, it is shown that the nonlocal scale parameters related to the size of the repetitive cell depends on the type of analysis, either buckling or vibration, when compared to the lattice results. As opposed to phenomenological nonlocal models, lattice-based continualized nonlocal models have constant length scale calibration. However, these continualized nonlocal models are constructed from nonlocal balance equations, whereas Eringen's nonlocal model keeps the locality of balance equations. This paper tries to open some new directions in generalizing nonlocal beam or plate models, from lattice mechanics. It is shown that some fundamental lattice plate interactions may be modeled at a larger scale by several nonlocal plate models, based on physical micromechanics arguments or introduced within mathematical properties.

\section{References}

1. Maugin, G.A.: Continuum Mechanics Through the Twentieth Century: A Concise Historical Perspective. Springer, Berlin (2013)

2. Krumhansl, J.A.: Generalized continuum field representation for lattice vibrations. In: Wallis, R.K. (ed.) Lattice Dynamics, pp. 627-634. Pergamon, London (1965)

3. Krumhansl, J.A.: Mechanics of Generalized Continua. Springer, New York (1968)

4. Rogula, D.: Influence of spatial acoustic dispersion on dynamical properties of dislocations. Bull. Acad. Pol. Sci. Sér. Sci. Tech. 13, 337-385 (1965)

5. Eringen, A.C.: Linear theory of micropolar elasticity. J. Math. Mech. 15, 909-923 (1966)

6. Eringen, A.C., Edelen, D.G.B.: On nonlocal elasticity. Int. J. Eng. Sci. 10, 233-248 (1972)

7. Kröner, E., Datta, B.K.: Nichtlokale elastostatik: Ableitung aus der gittertheorie. Z. Phys. 196, 203-211 (1966). (in German)

8. Kunin, I.A.: Model of elastic medium with simple structure and space dispersion. Prykl. Mat. Mekh. 30, 542-550 (1966)

9. Hencky, H.: Über die angenäherte Lösung von Stabilitätsproblemen im Raummittels der elastischen Gelenkkette. Der Eisenbau 11, 437-452 (1920). (in German)

10. Lagrange, J.L.: Recherches sur la nature et la propagation du son Miscellanea Philosophico-Mathematica Societatis Privatae Taurinensis I, 2rd Pagination, i- 112 (see also Euvres, Tome 1, pp 39-148) (1759) (in French)

11. Challamel, N., Wang, C.M., Elishakoff, I.: Nonlocal or gradient elasticity macroscopic models: a question of concentrated or distributed microstructure. Mech. Res. Commun. 71, 25-31 (2016)

12. Dell'Isola, F., Maier, G., Perego, U., Andreaus, U., Esposito, R., Forest, S.: The Complete Works of Gabrio Piola: Volume I Commented English Translation Advanced Structured Materials, vol. 38. Springer, Berlin (2014)

13. Dell'Isola, F., Andreaus, U., Placidi, L.: At the origins and in the vanguard of peridynamics non-local and higher-gradient continuum mechanics: an underestimated and still topical contribution of Gabrio Piola. Math. Mech. Solids 20, 887-928 (2015)

14. Eringen, A.C.: On differential equations of nonlocal elasticity and solutions of screw dislocation and surface waves. J. Appl. Phys. 54, 4703-4710 (1983)

15. Born, M., von Kármán, T.: On fluctuations in spatial grids. Phys. Z. 13, 297-309 (1912)

16. Challamel, N., Wang, C.M.: The small length scale effect for a non-local cantilever beam: a paradox solved. Nanotechnology 19, 345703 (2008)

17. Fernandez-Saez, J., Zaera, R., Loya, J.A., Reddy, J.N.: Bending of Euler-Bernoulli beams using Eringen's integral formulation: a paradox resolved. Int. J. Eng. Sci. 99, 107-116 (2016)

18. Zhang, Z., Wang, C.M., Challamel, N.: Eringen's length scale coefficient for vibration and buckling of nonlocal rectangular plates with simply supported edges. J. Eng. Mech. 141, 04014117 (2015)

19. Challamel, N., Lerbet, J., Wang, C.M., Zhang, Z.: Analytical length scale calibration of nonlocal continuum from a microstructured buckling model. Z. Angew. Math. Mech. 94, 402-413 (2014)

20. Challamel, N., Hache, F., Elishakoff, E., Wang, C.M.: Buckling and vibrations of microstructured rectangular plates considering phenomenological and lattice-based nonlocal continuum model. Compos. Struct. 149, 145-156 (2016)

21. Askes, H., Metrikine, A.V.: Higher-order continua derived from discrete media: continualisation aspects and boundary conditions. Int. J. Solids Struct. 42, 187-202 (2005) 
22. Tollenaere, H., Caillerie, D.: Continuous modeling of lattice structures by homogenization. Adv. Eng. Softw. 29, 699-705 (1998)

23. Andrianov, I.V., Awrejcewicz, J., Weichert, D.: Improved continuous models for discrete media. Math. Probl. Eng. (2010). doi:10.1155/2010/986242

24. Kruskal, M.D., Zabusky, N.J.: Stroboscopic perturbation for treating a class of nonlinear wave equations. J. Math. Phys. 5, 231-244 (1964)

25. Rosenau, P.: Dynamics of nonlinear mass-spring chains near the continuum limit. Phys. Lett. A 118, $222-227$ (1986)

26. Rosenau, P.: Dynamics of dense lattices. Phys. Rev. B 36, 5868 (1987)

27. Benjamin, T.B., Bona, J.L., Mahony, J.J.: Model equations for long waves in nonlinear dispersive systems. Philos. Trans. R. Soc. Lond. Ser. A Math. Phys. Sci. 272, 47-78 (1972)

28. Collins, M.A.: A quasicontinuum approximation for solitons in an atomic chain. Chem. Phys. Lett. 77, 342-347 (1981)

29. Kevrekidis, P.G., Kevrekidis, I.G., Bishop, A.R., Titi, E.S.: Continuum approach to discreteness. Phys. Rev. E 65, 046613 (2002)

30. Andrianov, I.V., Danishevs'kyy, V.V., Kalamkarov, A.L.: Vibration localization in one-dimensional linear and nonlinear lattices: discrete and continuum models. Nonlinear Dyn. (2012). doi:10.1007/s11071-012-0688-4

31. Giorgio, I., Della Corte, A., dell'Isola, F. , : Dynamics of 1D nonlinear pantographic continua. Nonlinear Dyn (2016). doi:10. 1007/s11071-016-3228-9

32. Ansari, R., Hemmatnezhad, M.: Nonlinear finite element analysis for vibrations of double-walled carbon nanotubes. Nonlinear Dyn. (2012). doi:10.1007/s11071-011-9985-6

33. Wang, Y., Li, F.M., Wang, Y.Z.: Nonlocal effect on the nonlinear dynamic characteristics of buckled parametric double-layered nanoplates. Nonlinear Dyn. (2016). doi:10.1007/s11071-016-2789-y

34. El Naschie, M.S.: Stress, Stability and Chaos in Structural Engineering: An Energy Approach. McGraw Hill, London (1991)

35. Challamel, N., Wang, C.M., Elishakoff, I.: Discrete systems behave as nonlocal structural elements: bending, buckling and vibration analysis. Eur. J. Mech. A Solid 44, 125-135 (2014)

36. Seide, P.: Accuracy of some numerical methods for column buckling. J. Eng. Mech. 101, 549-560 (1975)

37. Wang, C.T.: Discussion on the paper of "Salvadori M.G., Numerical computation of buckling loads by finite differences". Trans. ASCE 116, 629-631 (1951)

38. Wang, C.T.: Applied Elasticity. McGraw-Hill, New York (1953)

39. Santoro, R., Elishakoff, I.: Accuracy of the finite difference method in stochastic settings. J. Sound Vib. 291, 275-284 (2006)

40. Leckie, F.A., Lindberg, G.M.: The effect of lumped parameters on beam frequencies. Aeronaut. Q. 14, $224-240$ (1963)

41. Peddieson, J., Buchanan, G.R., McNitt, R.P.: Application of nonlocal continuum models to nanotechnology. Int. J. Eng. Sci. 41, 305-312 (2003)

42. Sudak, L.J.: Column buckling of multiwalled carbon nanotubes using nonlocal continuum mechanics. J. Appl. Phys. 94, 7281-7287 (2003)

43. Reddy, J.N.: Nonlocal theories for bending, buckling and vibration of beams. Int. J. Eng. Sci. 45, 288-307 (2007)

44. Reddy, J.N., Pang, S.D.: Nonlocal continuum theories of beams for the analysis of carbon nanotubes. J. Appl. Phys. 103, $023511(2008)$

45. Adali, S.: Variational principles for multi-walled carbon nanotubes undergoing buckling based on nonlocal elasticity theory. Phys. Lett. A 372, 5701-5705 (2008)

46. Adali, S.: Variational principles for transversely vibrating multiwalled carbon nanotubes based on nonlocal Euler-Bernoulli beam model. Nanoletters 9, 1737-1741 (2009)

47. Challamel, N., Zhang, Z., Wang, C.M., Reddy, J.N., Wang, Q., Michelitsch, T., Collet, B.: On non-conservativeness of Eringen's nonlocal elasticity in beam mechanics: correction from a discrete-based approach. Arch. Appl. Mech. 84, 1275$1292(2014)$

48. Zhang, Z., Wang, C.M., Challamel, N.: Eringen's length scale coefficient for buckling of nonlocal rectangular plates from microstructured beam-grid model. Int. J. Solids Struct. 51, 4307-4315 (2014)

49. Lu, P., Zhang, P.Q., Lee, H.P., Wang, C.M., Reddy, J.N.: Non-local elastic plate theories. Proc. R. Soc. A 463, 3225-3240 (2007)

50. Chakraverty, S., Behera, L.: Free vibration of rectangular nanoplates using Rayleigh-Ritz method. Phys. E Low Dimens. Syst. Nanostruct. 56, 357-363 (2014)

51. Phadikar, J.K., Pradhan, S.C.: Variational formulation and finite element analysis for nonlocal elastic nanobeams and nanoplates. Comput. Mater. Sci. 49, 492-499 (2010)

52. Adali, S.: Variational principles and natural boundary conditions for multilayered orthotropic graphene sheets undergoing vibrations and based on nonlocal elasticity. J. Theor. Appl. Mech. 49, 621-639 (2011)

53. Adali, S.: Variational principles for nonlocal continuum model for orthotropic graphene sheets embedded in an elastic medium. Acta Math. Sci. 32, 325-338 (2012) 